\title{
Right Temporoparietal Junction Underlies Avoidance of Moral Transgression in Autism Spectrum Disorder
}

\author{
Yang Hu, ${ }^{1,2,5 *}$ Alessandra M. Pereira, ${ }^{3 *}{ }^{\circledR}$ Xiaoxue Gao, ${ }^{5}$ Brunno M. Campos, ${ }^{3}$ Edmund Derrington, ${ }^{2,4}$ \\ Brice Corgnet, ${ }^{7}{ }^{\circledR}$ Xiaolin Zhou, ${ }^{1,5,6}$ Fernando Cendes, ${ }^{3}$ and ${ }^{\circledR}$ Jean-Claude Dreher ${ }^{2,4}$ \\ ${ }^{1}$ Key Laboratory of Applied Brain and Cognitive Sciences, School of Business and Management, Shanghai International Studies University, \\ Shanghai 201620, People's Republic of China, ${ }^{2}$ Institut des Sciences Cognitives Marc Jeannerod, CNRS, Neuroeconomics Lab 69675 Bron, France, \\ ${ }^{3}$ Neuroimaging Laboratory, School of Medical Sciences, The Brazilian Institute of Neuroscience and Neurotechnology, University of Campinas \\ (UNICAMP), Campinas 13083-970, Brazil, ${ }^{4}$ Université Claude Bernard Lyon 1, 69100 Villeurbanne, France, ${ }^{5}$ School of Psychological and Cognitive \\ Sciences, Peking University, Beijing 100871, People's Republic of China, ${ }^{6} \mathrm{PKU}-\mathrm{IDG} / \mathrm{McGovern}$ Institute for Brain Research, Peking University, \\ Beijing 100871, People's Republic of China, and ${ }^{7}$ EmLyon, 69130 Ecully, France
}

Autism spectrum disorder (ASD) is characterized by a core difference in theory-of-mind (ToM) ability, which extends to alterations in moral judgment and decision-making. Although the function of the right temporoparietal junction (rTPJ), a key neural marker of ToM and morality, is known to be atypical in autistic individuals, the neurocomputational mechanisms underlying its specific changes in moral decision-making remain unclear. Here, we addressed this question by using a novel fMRI task together with computational modeling and representational similarity analysis (RSA). ASD participants and healthy control subjects (HCs) decided in public or private whether to incur a personal cost for funding a morally good cause (Good Context) or receive a personal gain for benefiting a morally bad cause (Bad Context). Compared with HC, individuals with ASD were much more likely to reject the opportunity to earn ill gotten money by supporting a bad cause than were HCs. Computational modeling revealed that this resulted from heavily weighing benefits for themselves and the bad cause, suggesting that ASD participants apply a rule of refusing to serve a bad cause because they evaluate the negative consequences of their actions more severely. Moreover, RSA revealed a reduced rTPJ representation of the information specific to moral contexts in ASD participants. Together, these findings indicate the contribution of rTPJ in representing information concerning moral rules and provide new insights for the neurobiological basis underpinning moral behaviors illustrated by a specific difference of rTPJ in ASD participants.

Key words: autism; decision-making; fMRI; moral

Significance Statement

Previous investigations have found an altered pattern of moral behaviors in individuals with autism spectrum disorder (ASD), which is closely associated with functional changes in the right temporoparietal junction (rTPJ). However, the specific neurocomputational mechanisms at play that drive the altered function of the rTPJ in moral decision-making remain unclear. Here, we show that ASD individuals are more inflexible when following a moral rule although an immoral action can benefit themselves, and experience an increased concern about their ill-gotten gains and the moral cost. Moreover, a selectively reduced rTPJ representation of information concerning moral rules was observed in ASD participants. These findings deepen our understanding of the neurobiological roots that underlie atypical moral behaviors in ASD individuals.

Received May 25, 2020; revised 0ct. 27, 2020; accepted 0ct. 28, 2020.

Author contributions: A.M.P., F.C., and J.-C.D. designed research; A.M.P., B.M.C., F.C., and J.-C.D. performed research; Y.H. and X.G. analyzed data; Y.H., E.D., B.C., X.Z., F.C., and J.-C.D. wrote the paper.

*Y.H. and A.M.P. contributed equally to this study.

J.-C.D. was funded by the IDEX-LYON from Université de Lyon (project INDEPTH) within the Program Investissements d'Avenir (ANR-16-IDEX-0005) and by the LABEX CORTEX (ANR-11-LABX-0042) of Universite de Lyon within the program Investissements d'Avenir (ANR-11-IDEX-007) operated by the French National Research Agency; and by grants from the Agence Nationale pour la Recherche and the National Science Foundation in the Collaborative Research in Computational Neuroscience (CRCNS) program (ANR \#16-NEUC0003-01) and from Fondation de France (\#89590). F.C. was funded by the São Paulo Research Foundation (FAPESP Grant \#2013/07559-3) of Brazil. Y.H. was funded by the China Postdoctoral Science Foundation
(2019M660007). X.G. and X.Z. were supported by the National Basic Research Program of China $(973$ Program: 2015CB856400) and the National Natural Science Foundation of China (Grants 91232708, 31170972, 31630034, and 71942001). X.G. was supported by the China Postdoctoral Science Foundation (Grant 2019M650008) and the National Natural Science Foundation of China (Grant 31900798). We thank the staff of the Imaging Center of the University of Campinas for helpful assistance with data collection for the fMRI study.

The authors declare no competing financial interests.

Correspondence should be addressed to Jean-Claude Dreher at dreher@isc.cnrs.fr.

https://doi.org/10.1523/JNEUROSCI.1237-20.2020

Copyright @ 2021 the authors 


\section{Introduction}

Autism spectrum disorder (ASD) is a complex neurodevelopment disorder with evident impairments in social interaction, communication, and interpersonal relationships (American Psychiatric Association, 2013), which are critically dependent on theory-of-mind (ToM) ability (Young et al., 2007; Margoni and Surian, 2016). These social problems are also associated with atypical moral cognition. Indeed, individuals with ASD have difficulties in evaluating the moral appropriateness of actions in terms of the intentions of the protagonists in hypothetical scenarios (Moran et al., 2011; Buon et al., 2013; Fadda et al., 2016). ASD individuals show differences in moral behaviors that lead to real consequences. For example, they are less sensitive to observation by others while making charitable decisions (Izuma et al., 2011).

Previous neuroimaging studies of healthy subjects lay a crucial foundation for improving our understanding of the neural basis underlying the atypical morality of ASD individuals. One of the key regions is the right temporoparietal junction (rTPJ), which is not only the hub of the ToM network (Schurz et al., 2014; Schaafsma et al., 2015), but is also well known for its crucial contribution to moral judgments (Young et al., 2007) and moral decisions involving tradeoffs between self-interest and other's welfare (Morishima et al., 2012; Tusche et al., 2016). Importantly, prior fMRI studies have also shown an atypical rTPJ activation in ASD cohorts compared with healthy control subjects (HCs) in a variety of social tasks that critically depend on ToM ability, such as processing naturalistic social situations (Pantelis et al., 2015), perceiving biological motion (Kana et al., 2009), or mentalizing about someone else (Lombardo et al., 2011). More relevantly, rTPJ in ASD individuals did not display reliable neural patterns that distinguish intentional harm from accidental harm (Koster-Hale et al., 2013). While these studies provide direct evidence of a ToM-related dysfunction of rTPJ in ASD individuals, the specific rTPJ dysfunction that drives atypical moral behaviors in ASD individuals remains largely unknown.

To address this question, we used a novel paradigm in an fMRI study where high-functioning ASD participants and HCs decided whether to accept or reject a series of offers. In particular, we independently manipulated two factors [i.e., Audience (whether decisions were made in public or private) and Moral Context (whether the offer involves a tradeoff between a personal financial loss and a charity donation, or between a personal financial gain and a donation to a morally bad cause)]. Moreover, the payoffs for participants and the associations varied across different trials in an orthogonal manner.

Combining computational modeling (Crockett, 2016; Konovalov et al., 2018) and multivariate-based representational similarity analyses (RSAs; Kriegeskorte et al., 2008), the present design allowed us to directly test two predictions about different aspects of atypical moral behaviors in ASD individuals and their critical association with rTPJ dysfunction. The first prediction concerned social reputation (Frith and Frith, 2011), namely, how individuals care about their self-image in other's eyes. Evidence has shown that while making prosocial decisions, ASD participants show difficulties in sustaining a social reputation, which requires mentalizing ability (Izuma et al., 2011). Thus, compared with the HC group, ASD participants would show less distinction between their moral decisions made in public and in private. This would be associated with a reduced rTPJ engagement of representing information concerning social reputation, in the presence or absence of an audience.
Table 1. Summary of clinical measures in two groups

\begin{tabular}{|c|c|c|}
\hline & ASD & $\mathrm{HC}$ \\
\hline IQ: total ${ }^{\mathrm{a}}$ & $100.0 \pm 10.0$ & $105.0 \pm 8.9$ \\
\hline IQ: verbal ${ }^{\mathrm{a}}$ & $103.2 \pm 9.9$ & $103.2 \pm 9.2$ \\
\hline IQ: execution ${ }^{\mathrm{a}}$ & $106.7 \pm 12.4$ & $106.7 \pm 11.5$ \\
\hline ADI-R: social & $21.0 \pm 5.2$ & \\
\hline ADI-R: communication & $14.0 \pm 4.5$ & \\
\hline ADI-R: repetitive & $6.7 \pm 1.7$ & \\
\hline
\end{tabular}

IQ, Intelligence quotient.

a IQ was measured by Wechsler Intelligence Scale for Children (WISC). Data from three HCs were missing

Our second hypothesis was inspired by studies of moral judgments that reveal autistic individuals tend to judge moral culpability more often in terms of consequences (Moran et al., 2011; Fadda et al., 2016; Salvano-Pardieu et al., 2016), and often overevaluate the negative moral consequences (Moran et al., 2011; Bellesi et al., 2018). Hence, it was possible that compared with HCs, ASD participants would display increased aversion to the consequences of an immoral action and therefore reject more offers that earn themselves morally tainted profits. We further explored whether such behavioral differences could be explained by a reduced rTPJ representation of information concerning moral contexts in ASD participants.

\section{Materials and Methods}

Participants

A total of 48 participants were recruited for the present fMRI experiment. Specifically, 20 individuals with ASD (4 females; mean age, $17.0 \pm 3.0$ years; age range, $14-24$ years; 3 left handed) were recruited via those who attended psychiatric and pediatric neurology clinics as outpatients and fulfilled the inclusion criteria. Twenty-eight HCs (10 females; mean age, $18.9 \pm 3.0$ years; age range, $14-25$ years; 1 left handed) were recruited from the local community via fliers. Diagnoses of ASD were performed by a clinical pediatric neurologist according to the Autism Diagnostic Interview-revised (ADI-R; Table 1, all clinical tests describing the two samples). There were no significant between-group differences in gender $\left(\chi(1)^{2}=1.395, p=0.238\right)$ and IQ (total: $t_{(43)}=-1.795, p=$ 0.080 ; verbal: $t_{(43)}=-1.379, p=0.175$; execution: $t_{(43)}=-1.421$, $p=0.162$ ), except that ASD participants were slightly younger than HC participants $\left(t_{(46)}=-2.121, p=0.039\right)$.

The study was performed at the Imaging Center of the University of Campinas and approved by the local ethics committee (https:// plataformabrasil.saude.gov.br; reference \#CAAE 02388012.5.0000.5404; approved ethical statement \#1904090). All experimental protocols and procedures were conducted in accordance with the institutional review board guidelines for experimental testing and complied with the latest revision of the Declaration of Helsinki.

Experimental design and task

We adopted a $2 \times 2$ within-subject design with a novel paradigm (but see Obeso et al., 2018; Qu et al., 2019). Specifically, participants decided whether to accept or reject a series of offers consisting of a personal profit or loss and a donation to a certain association, either in the absence or presence of an audience (i.e., Audience: Private vs Public; see Procedure for details). In half of the trials, participants were confronted with offers involving a monetary loss for themselves but a financial gain to a local charity, "The Child Hope Campaign" (www.redeglobo.globo. $\mathrm{com} /$ criancaesperanca), which supports the education of children and adolescents in Brazil. In the other trials, participants considered offers that comprised a monetary gain for themselves but also a financial gain benefiting a morally bad cause, "No Dogs and Cats", which aims to clean the street by exterminating street animals. In other words, we manipulated moral contexts (i.e., Good vs Bad) according to the cause involved in offers. In total, the present design yielded four experimental conditions, Public $\mathrm{Good}_{\mathrm{G}}$, Public $\mathrm{Bad}_{\mathrm{Bad}}$, Private ${ }_{\mathrm{Good}}$, and Private ${ }_{\mathrm{Bad}}$. Crucially, participants were informed that their decisions 


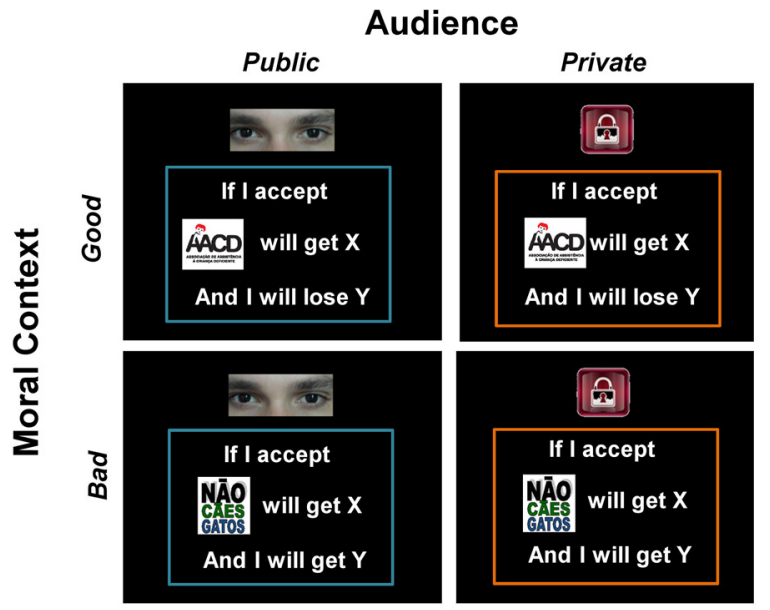

B
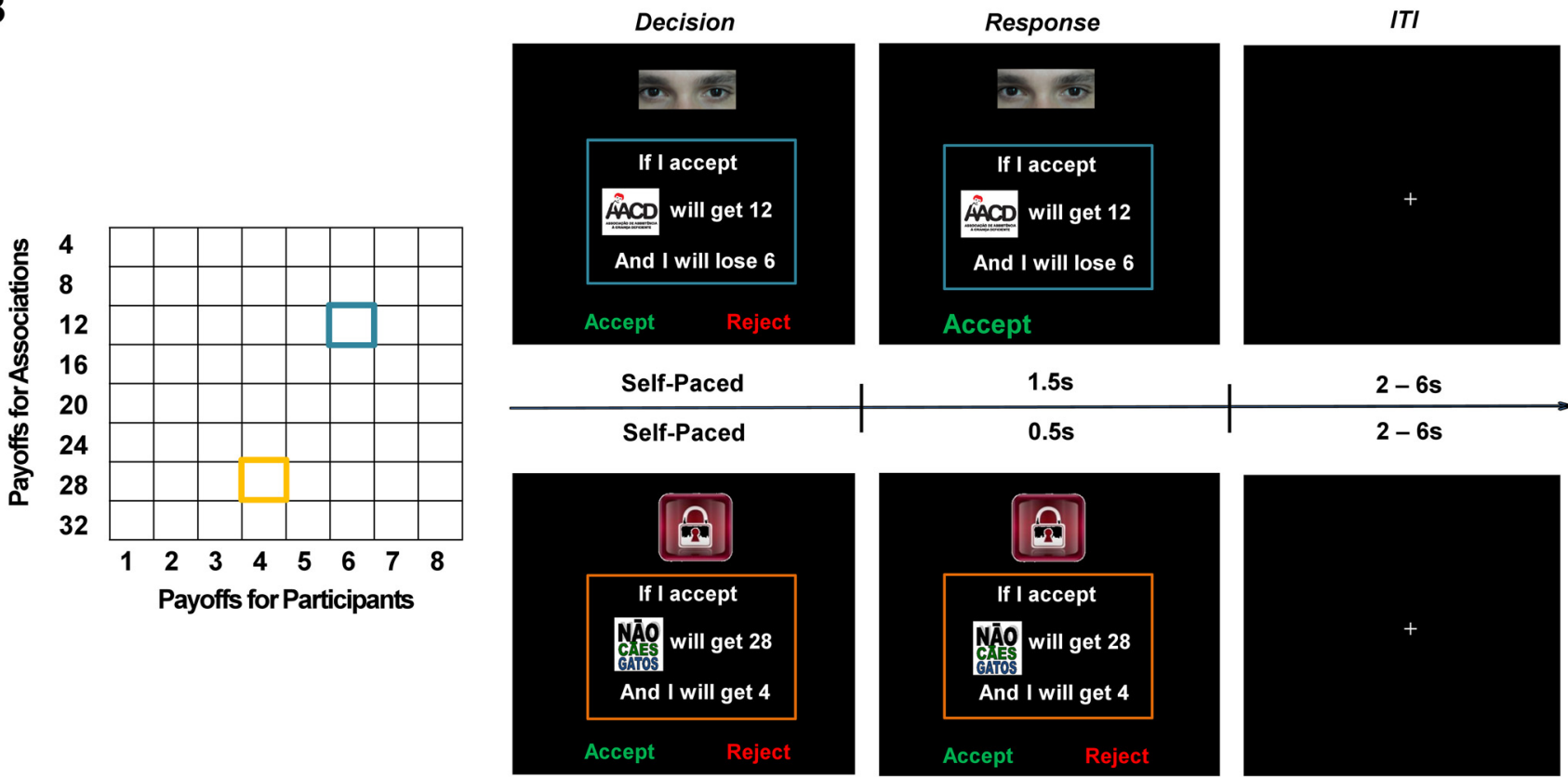

$2-6 s$
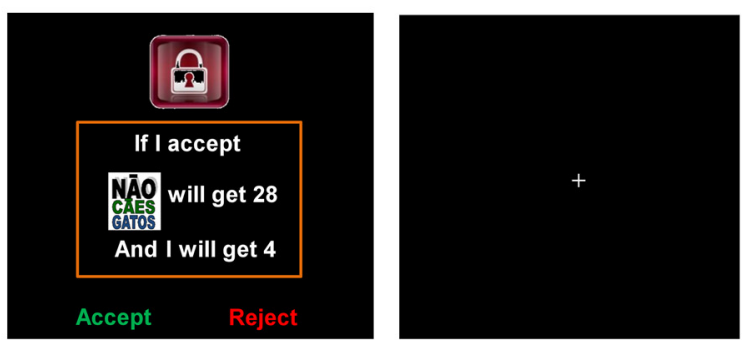

Figure 1. Illustration of experimental design and trial procedure. $A$, We used a $2 \times 2$ within-subject design by independently manipulating Audience (Private or Public) and Moral Context (Good or Bad), which yielded four experimental conditions (i.e., Publi $\mathrm{G}_{\mathrm{Good}}$, Public $_{\text {Bad, }}$ Private $\mathrm{Good}_{\mathrm{d}}$ and Private ${ }_{\mathrm{Bad}}$ ). The Public condition was indicated by the picture of "eyes," and the Private condition was indicated by the picture of a "lock." The Good Context involved a tradeoff between personal losses and benefits for a charity, whereas in the Bad Context participants traded personal benefits against benefits for a morally bad cause. $\boldsymbol{B}$, Monetary payoffs (in Brazilian Real) for participants (8 levels: from 1 to 8 , in steps of 1 ) and the association (8 levels: from 4 to 32 , in

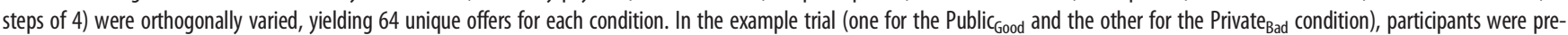
sented with an offer and decided whether to accept or reject the offer with no time limit. If they accepted the offer, both parties involved (i.e., the participant and the association) might undergo the financial consequences as proposed. If they rejected the offer, neither party would profit. In the Private condition, once a response was made, the screen was unchanged for $0.5 \mathrm{~s}$ to keep the chosen option private. In the Public condition, the chosen option was highlighted with a larger font and the nonchosen option disappeared, this lasted slightly longer (1.5 $\mathrm{s}$ ) to further emphasize the presence of a witness. Each trial was ended with an intertrial interval (ITI) showing a jittered fixation $(2.5 \sim 6.5 \mathrm{~s})$.

could have real consequences. Thus, if participants accepted the offer in the Good context, they would lose a certain amount of money and the charity would be paid. If they do so in the Bad context, they would earn the money and the bad cause would also be paid. However, if participants rejected the offer, neither they nor the involved association would gain or lose any money (Fig. 1). Participants were also informed that all trials (decisions) were independent from each other so that the incentive consequences would not accumulate across the experiment. Only one trial would be randomly selected and paid at the end of the experiment.

One key aspect of the present design was that we varied the monetary stakes for the participants and the associations independently across trials within each condition. Personal payoffs (i.e., profits or losses) ranged from 1 to 8 in steps of 1 (unit, Brazillian Real; 1 Brazilian Real $=\sim 0.2$ US Dollars). Donations to both associations ranged from 4 to 32 in the steps of 4 . The personal payoff and the donation were orthogonal, which led to 64 different offers. Each offer appeared only once in each condition and thus summed up to 256 trials in total.

The functional scanning comprised four runs of 64 trials. Each moral context was assigned to either the first or the second of two runs. Each run consisted of two blocks, which included 32 trials presenting unique offers in either the Private or the Public condition. The order of runs involving Good/Bad moral contexts and Public/Private blocks were counterbalanced across participants. The trial order was randomized within each block. For each trial, participants were presented with the decision screen consisting of the payoff information for the participant (monetary gain or loss), and the association indicated by the corresponding symbol. The cue that signaled whether it was a Public (a picture of eyes) or a Private (i.e., a picture of a padlock) trial was also shown on the same screen. Here a cue of being watched was used as previous studies have consistently shown that it 
A

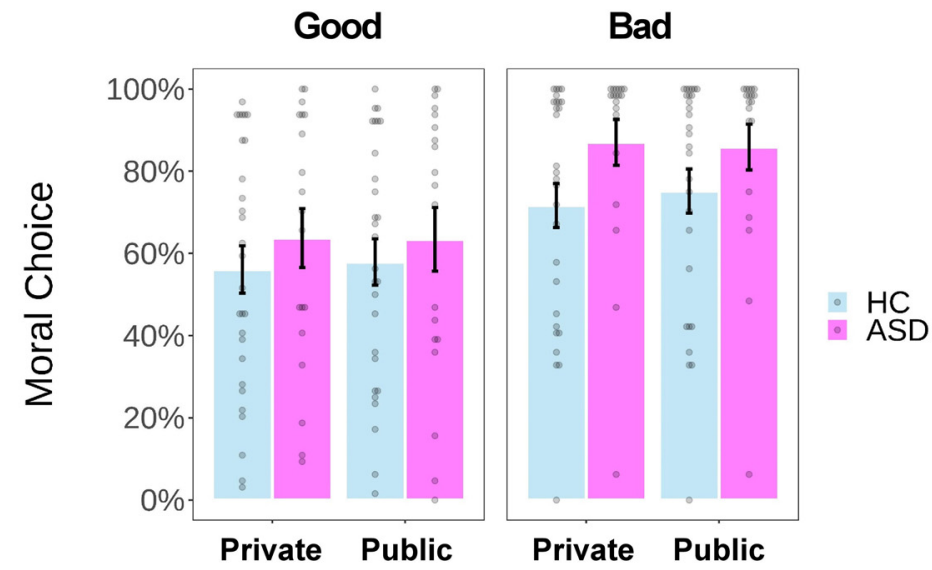

B
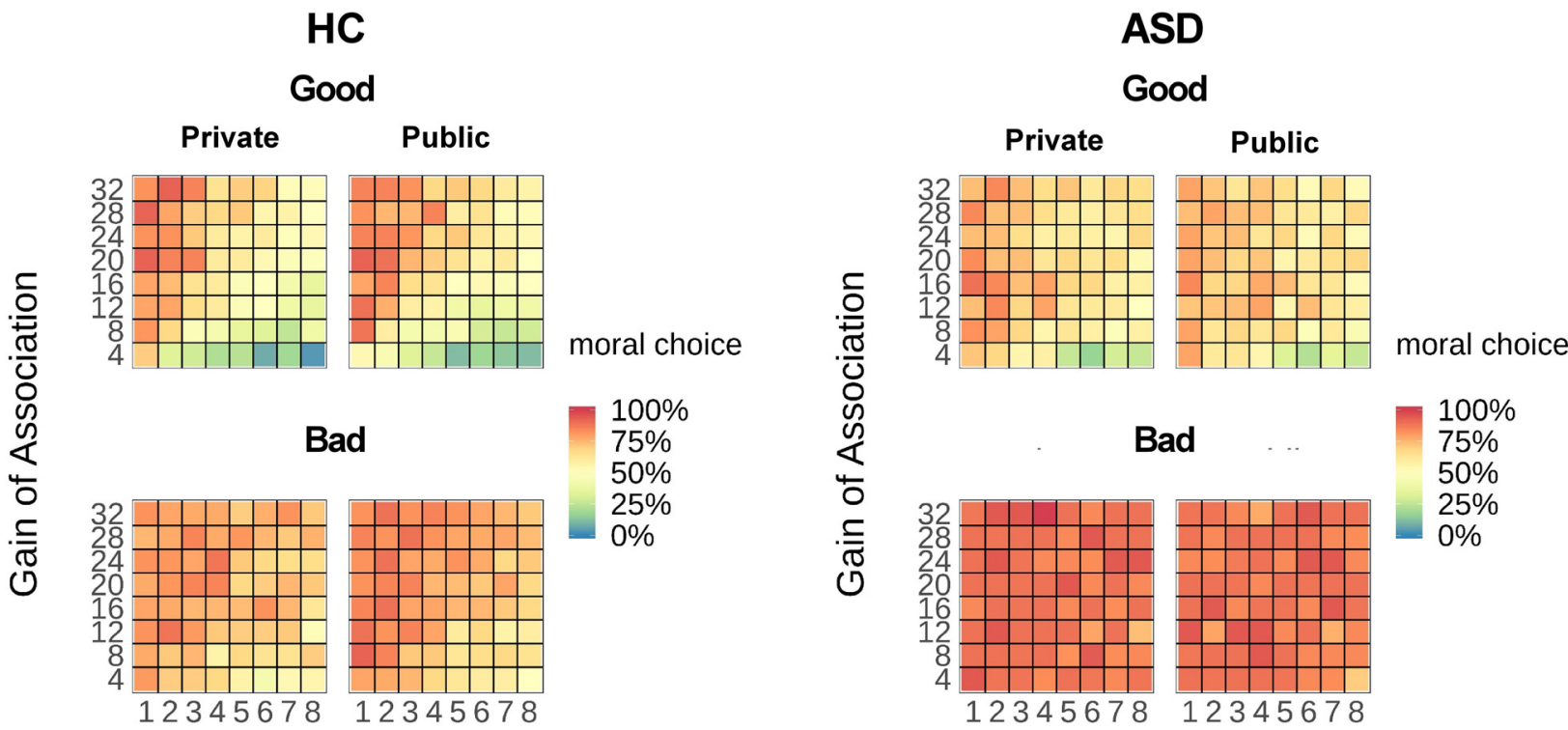

\section{Gain/Loss of Participant}

\section{Gain/Loss of Participant}

Figure 2. Results of choice behavior. A, Rate of choosing the moral option as a function of group (ASD or HC), reputation (Private or Public), and context (Good or Bad). $\boldsymbol{B}$, Heat map of the mean proportion (percentage) of moral choices as a function of payoffs (monetary units) for participants and for associations in each experimental condition for each group. Each dot represents the data of a single participant. Error bars represent the SEM.

influences individuals' behaviors (Haley and Fessler, 2005; Izuma et al., 2008). Participants decided whether to accept or reject the offer by pressing the corresponding button on the button box with the right index or middle finger at their own pace. In the Private condition, once a response was made, the screen was unchanged for $0.5 \mathrm{~s}$ to keep the chosen option private. In the Public condition, the chosen option was highlighted with a larger font, and the nonchosen option disappeared, which lasted slightly longer (1.5 s) to further emphasize the presence of a witness (Qu et al., 2019). This was followed by a uniformly jittered fixation $(2.5-6.5 \mathrm{~s})$, which ended the trial.

All visual stimuli were presented using Presentation version 14 (Neurobehavioral Systems) back-projected on a screen outside the scanner, using a mirror system attached to the head coil.

\section{Procedure}

On the day of scanning, participants (and their legal guardians when necessary) first signed the written informed consent and then were given the instructions. After that, they completed a series of comprehension questions to ensure that they fully understood the task. Importantly, they met with an independent audience and were informed that this person would sit in the control room to witness their choices in some trials (i.e., in the Public condition) during the experiment. In the scanner, participants completed a practice session to get familiar with the paradigm and the response button. The scanning part consisted of four functional runs lasting $\sim 35 \mathrm{~min}$, which was followed by a 6 min structural scan. After that, participants indicated their liking for each association on an 11 point Likert scale ( 0 indicated "dislike very much," 10 indicated "like very much"). Finally, participants were debriefed, paid, and thanked.

\section{Data acquisition}

The imaging data were acquired on a 3 tesla Philips Achieva MRI system with a 32-channel head coil (Best) at the Imaging Center of University of Campinas. Functional data were acquired using T2*-weighted echoplanar imaging (EPI) sequences using a BOLD contrast $(\mathrm{TR}=2000 \mathrm{~ms}$; $\mathrm{TE}=30 \mathrm{~ms}$; flip angle $=90^{\circ}$; slice thickness $=3 \mathrm{~mm}$ without gap; matrix $\left.=80 \times 80 ; \mathrm{FOV}=240 \times 240 \mathrm{~mm}^{2}\right)$ in 40 axial slices. Slices were 
Table 2. Results of mixed-effect logistic regressions predicting moral choices

\begin{tabular}{|c|c|c|c|c|c|}
\hline & $\mathrm{All}^{\mathrm{b}}$ & Good $^{b}$ & $\mathrm{Bad}^{b}$ & Bad: private & Bad: public \\
\hline Intercept & $0.63(0.39)$ & $0.54(0.53)$ & $2.64^{* * *}(0.81)$ & $2.57^{* *}(0.84)$ & $3.26^{\text {**** }}(0.88)$ \\
\hline Group & $0.86(0.64)$ & $1.31(0.86)$ & $3.41^{*}(1.42)$ & $4.16^{* *}(1.53)$ & $2.32(1.44)$ \\
\hline Audience & $0.11(0.08)$ & $0.15(0.10)$ & $0.27^{* *}(0.10)$ & & \\
\hline Moral context & $0.95^{* * *}(0.08)$ & & & & \\
\hline Group $\times$ audience & $-0.17(0.13)$ & $-0.23(0.16)$ & $-0.44^{*}(0.22)$ & & \\
\hline Group $\times$ moral context & $0.89^{* * *}(0.15)$ & & & & \\
\hline Audience $\times$ moral context & $0.09(0.12)$ & & & & \\
\hline Group $\times$ Audience $\times$ Moral context & $-0.11(0.21)$ & & & & \\
\hline Payoff for oneself ${ }^{a, b}$ & & $-0.99^{* * * *}(0.04)$ & $-0.46^{* * * *}(0.05)$ & $-0.39^{\text {***** }}(0.07)$ & $-0.56^{* * *}(0.07)$ \\
\hline Payoff for association ${ }^{\mathrm{a}, \mathrm{b}}$ & & $0.83 * * *(0.04)$ & $.33^{* * * *}(0.05)$ & $0.34^{* * * *}(0.06)$ & $0.35^{* * * *}(0.07)$ \\
\hline $\mathrm{Age}^{\mathrm{a}}$ & $0.19(0.32)$ & $0.50(0.43)$ & $0.26(0.70)$ & $0.23(0.70)$ & $0.12(0.73)$ \\
\hline $\mathrm{AlC}$ & $10,501.0$ & 4340.7 & 3148.7 & 1649.7 & 1551.1 \\
\hline $\mathrm{BIC}$ & $10,574.8$ & 4394.1 & 3202.2 & 1685.6 & 1587.1 \\
\hline$N$ (Observation) & 11,823 & 5912 & 5911 & 2948 & 2963 \\
\hline$N$ (Participant) & 47 & 47 & 47 & 47 & 47 \\
\hline
\end{tabular}

Values are the mean (SE), unless otherwise indicated. Reference levels were set as follows: Group, HCs; Audience, private; Moral context, good. The table also shows goodness-of-fit statistics. BIC, Bayesian information criterion.

${ }^{a}$ We standardized these variables for the analyses.

${ }^{b}$ These variables were added as covariates only when the regressor Association (and its interaction) was not in the regression model, as the regressor "payoff for oneself" qualitatively covaried with Association, which might cause the collinear issue.

$* p<0.05, * * p<0.01, * * * p<0.001$

A
HC

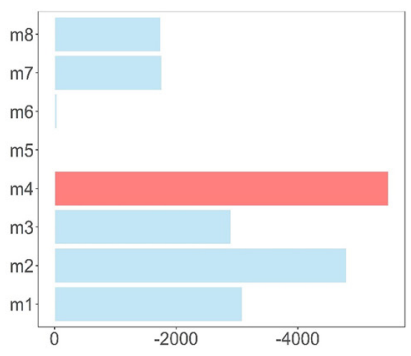

delta log model evidence (LOOIC)

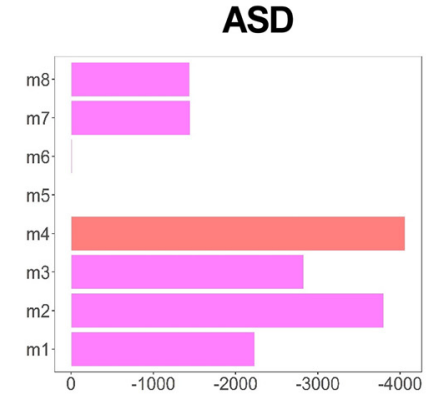

delta log model evidence (LOOIC)
B

Private

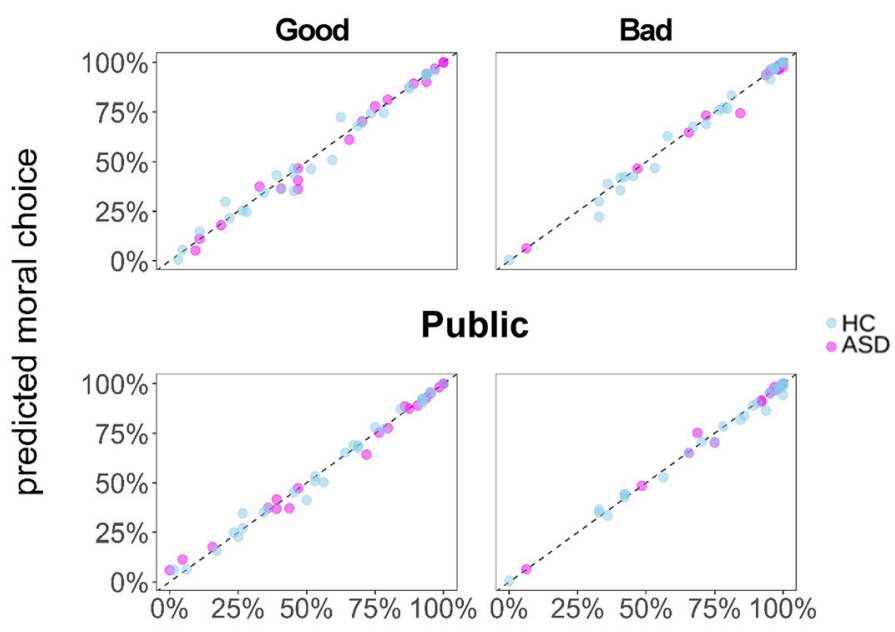

actual moral choice

Figure 3. Model comparison and validation. $\boldsymbol{A}$, Bayesian model evidence. Model evidence (relative to the model with the worst accuracy of out-of-sample prediction; i.e., model 5) clearly favors model 4 (m4). Lower (i.e., more negative) LOOIC scores indicate a better model. B, Posterior predictive check of the winning model. Each dot represents the data of a single participant. For each participant, we calculated the mean of the predicted proportion of moral choice (\%; $y$-axis) by averaging moral choices generated using the whole posterior distribution of estimated parameters specific to that participant based on the winning model. Regardless of experimental conditions, these dots almost fell on the diagonal, indicating that the winning model captured the actual behaviors of all participants in this task. axially oriented along the anterior commissureposterior commissure plane and acquired in an ascending order. A high-resolution structural T1-weighted image was also collected for every participant using a 3D MRI sequence (TR = $7 \mathrm{~ms}$; $\mathrm{TE}=3.2 \mathrm{~ms}$; flip angle $=8^{\circ}$; slice thickness $=1 \mathrm{~mm} ;$ matrix $=240 \times 240 ; \mathrm{FOV}=240 \times$ $240 \mathrm{~mm}^{2}$ ).

\section{Statistical analyses}

One ASD participant was excluded from behavioral analyses because of the invariant response pattern (i.e., rejecting all trials in the task). After checking the preprocessed fMRI data, we excluded two more HC participants [one because half of the scanning data was lost because of a technical reason, the other for excessive head motion (i.e., $>3$ $\mathrm{mm}$ ) in two of four runs] and one more ASD participant [because of excessive head motion (i.e., $>$ $5 \mathrm{~mm}$ ) in three of four functional runs]. Thus, 26 HC participants and 18 ASD participants were included for the fMRI analyses.

\section{Behavioral analyses}

All behavioral analyses were conducted using $\mathrm{R}$ (http://www.r-project.org/; R Core Team, 2014). All reported $p$ values are two-tailed and $p<0.05$ was considered statistically significant. Data visualization was performed via the "ggplot2" package (Wickham, 2016). We excluded trials with either extremely fast responses (i.e., $<200 \mathrm{~ms}$ ) or extremely slow responses (i.e., exceeding 3 SDs of the individual mean decision time) from both the behavioral analyses and the model-based analyses. The percentage of trials excluded because of the criteria of decision time was $1.63 \%$ for the HC group and $1.89 \%$ for the ASD group.

For ease of interpretation, we defined the moral choices as those in which the participant accepted offers in the Good context or rejected offers in the Bad context. We performed the repeated mixed-effect logistic regression predicting the moral choice by the glmer function in "lme4" package (Bates et al., 2013), with Group 
(dummy variable; reference level; same below), Audience (dummy variable; reference level: private; same below), Moral Context (dummy variable; reference level: good; same below), and their interactions (i.e., three two-way interactions and one three-way interaction) as the fixed-effect predictors. We also incorporated age as a covariate in the analyses to rule out its possible confounding effect. We included random-effects predictors that allowed varying intercepts across participants. For the statistical inference on each predictor, we performed the type II Wald $\chi^{2}$ test on the model fits by using the Anova function in "car" package (Fox et al., 2016). Once the interactions were detected, we ran post hoc regressions on the subset of data given the different groups and then conditions. We reported the odds ratio (OR) as an index of effect size of each predictor on moral choices.

We also performed mixed-effects linear regression analyses on the log-transformed decision time (Anderson-Darling normality test: $\mathrm{A}=431.33, p<0.001$ ) with the lmer function in the lme4 package, with the same fixed-effects predictors, random-effects predictors, and covariates as for the choice analyses. In addition, we also controlled the effect of specific decision (dummy variable; reference level: moral choice) in the regression model. We followed the procedure recommended by Luke (2017) to obtain the statistics for each predictor by applying the Satterthwaite approximations on the restricted maximum likelihood model (REML) fit via the "ImerTest" package (Luke, 2017). In addition, we reported the standardized coefficient $\left(b_{z}\right)$ as an index of the effect size of each predictor on decision time together with other continuous dependent measures (e.g., rating, parameter estimates) using "EMAtools" (https://cran.r-project.org/web/packages/EMAtools/) and the "lm. $\beta$ " package (https://cran.r-project.org/web/packages/lm.beta/) for mixed effects and simple linear regression models, respectively.

\section{Computational modeling}

To examine how participants evaluated payoffs of each party and integrated them into a subjective value (SV), we compared the following eight models with different utility functions characterizing participants' choices.

Model 1 was adapted from a recent study on moral decision-making by Crockett et al. $(2014,2017)$, which could be formally represented as follows:

$$
\mathrm{SV}\left(M_{S}, M_{O}\right)=\left\{\begin{array}{c}
-(\alpha-q * \theta) * M_{S}+(1-\alpha+q * \theta) * M_{O} \text { if Good } \\
(\alpha-q * \theta) * M_{S}-(1-\alpha+q * \theta) * M_{O} \text { if Bad, }
\end{array}\right.
$$

where SV denotes the SV of the given trial if the participant chooses to accept. For rejection trials, SV is always 0 given the rule of the task (i.e., neither beneficiaries would gain the money; same for all models). $M_{s}$ and $M_{o}$ represent the payoff (gain or loss) for oneself and payoffs donated to the corresponding association. $\alpha(0<\alpha<1)$ is the unknown parameter of social preference that arbitrates the relative weight on the payoff for the participant in the decision. $\theta(0<\theta<1)$ is the unknown parameter characterizing the audience effect, which is modulated by an indicator function $q$ ( 0 for private, 1 for public; same below). This model assumes that the subjective value was computed as a weighted summation of personal payoffs and payoffs donated to the association, and that people cared less about their own payoffs but increased the weights on the benefits donated to the association in public (vs private). Model 2 was similar to Model 1 except that it adopted two separate $\alpha$ values depending on the moral context in that trial.

Model 3 has a logic similar to that of Model 1 and was built on studies adopting a donation task (Lopez-Persem et al., 2017; Qu et al., 2020), as follows:

$$
\mathrm{SV}\left(M_{S}, M_{O}\right)=\left\{\begin{array}{c}
-(\alpha-q * \theta) * M_{S}+(\beta+q * \theta) * M_{O} \text { if Good } \\
(\alpha-q * \theta) * M_{S}+(\beta-q * \theta) * M_{O} \text { if Bad, }
\end{array}\right.
$$

where $\alpha$ and $\beta$ are unknown parameters that capture the weight of the payoff for either the participant or the association involved in the trial $(-20<\alpha, \beta<20)$. Again, $\theta(0<\theta<10)$ describes the audience effect, which is represented by the indicator function $q$. Model 4 was similar to Model 3 except that it adopted two separate pairs of $\alpha$ and $\beta$ according to the association involved in that trial (i.e., good cause or the bad cause).

Models 5-8 were established on the basis of the Fehr-Schmidt model (Fehr and Schmidt, 1999), as follows:

$$
\begin{gathered}
\operatorname{SV}\left(M_{S}, M_{O}\right) \\
=\left\{\begin{array}{c}
-M_{S}-\alpha * \max \left(M_{O}+M_{S}, 0\right)-\beta * \max \left(-M_{S}-M_{O}, 0\right) \text { if Good } \\
M_{S}-\alpha * \max \left(M_{O}-M_{S}, 0\right)-\beta * \max \left(M_{S}-M_{O}, 0\right) \text { if } \mathrm{Bad},
\end{array}\right.
\end{gathered}
$$

where $\alpha$ and $\beta$ measure the degree of aversion to payoff inequality in disadvantageous and advantageous situations respectively (i.e., how participants dislike that they themselves gained less/more than the association; $0<\alpha, \beta<5$ ). Among them, Model 5 adopted a fixed pair of $\alpha$ and $\beta$ values in all four conditions. Model 6 and Model 7 took different pairs of $\alpha$ and $\beta$ values either in terms of the audience or the moral context. Model 8 assumed that people showed distinct advantageous and disadvantageous inequality aversion that changed in each of the four conditions.

Given the softmax rule, we could estimate the probability of making a moral choice (i.e., accept in the Good context or reject in the Bad context) as below:

$$
\mathrm{p}\left(\mathrm{SV}_{\text {moral }}\right)=\frac{e^{\tau \mathrm{S} \mathrm{V}_{\text {moral }}}}{e^{\tau \mathrm{S} \mathrm{V}_{\text {moral }}}+e^{\tau \mathrm{SV} \text { immoral }}},
$$

where $\tau$ refers to the inverse softmax temperature $(0<\tau<10)$, which denotes the sensitivity of an individual's behavior to the difference in SV between moral and immoral choices.

We leveraged a hierarchical Bayesian analysis (HBA) approach (Gelman et al., 2014) to fit all the above candidate models via the "hBayesDM" package (Ahn et al., 2017). In general, HBA has several advantages over the traditional maximal likelihood estimation approach such that it could provide more stable and accurate estimates, and estimate the posterior distribution of both the group-level and individuallevel parameters simultaneously (Ahn et al., 2011). The hBayesDM package performs a full Bayesian inference and provides actual posterior distribution using a Markov chain Monte Carlo (MCMC) sampling manner through the Stan language (Gelman et al., 2015). Conforming to the default setting in this package, we assumed that the individual-level parameters were drawn from a group-level normal distribution: individual-level parameters $\sim$ normal $(\mu, \sigma)$. We fit each candidate model with four independent MCMC chains using 1000 iterations after 2000 iterations for the initial algorithm warmup per chain that results in 4000 valid posterior samples. The convergence of the MCMC chains was assessed through Gelman-Rubin R-hat Statistics (Gelman and Rubin, 1992).

For model comparisons, we computed the leave-one-out information criterion (LOOIC) score for each candidate model (Bault et al., 2015). LOOIC score provides the estimate of out-of-sample predictive accuracy in a fully Bayesian way, which makes it more reliable than the point estimate information criterion [e.g., Akaike information criterion (AIC)]. By convention, the lower LOOIC score indicates better out-ofsample prediction accuracy of the candidate model. A difference score of 10 on the information criterion scale is considered decisive (Burnham and Anderson, 2004). We selected the model with the lowest LOOIC as the winning model for subsequent analysis of key parameters. A posterior predictive check was additionally implemented to examine the absolute performance of the winning model. In other words, we tested whether the prediction of the winning model could capture the actual behaviors. In terms of the actual trial-wise stimuli sequences, we used each individual's joint posterior MCMC samples (i.e., 4000 times) to generate new choice datasets correspondingly (i.e., 4000 choices per trial per participant). Then we calculated the mean proportion of moral choices of each experimental condition in these new datasets for each subject, respectively. We performed a Pearson correlation to examine to what degree the predicted proportion of moral choice correlated with the actual proportion across individuals in each condition, respectively. 
A

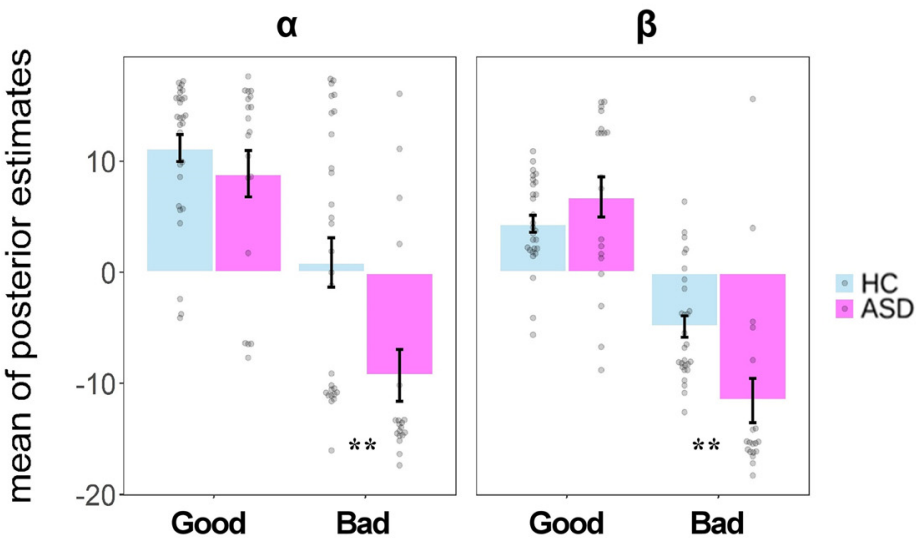

B

Good

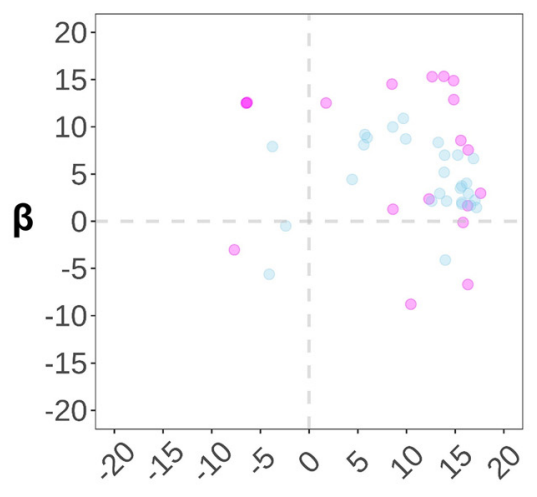

Bad

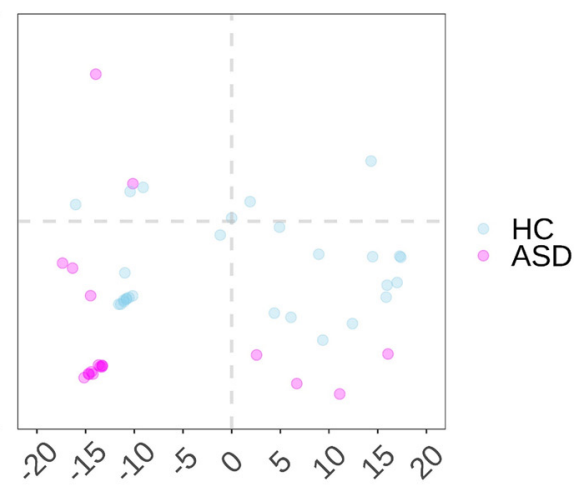

$\boldsymbol{\alpha}$

Figure 4. Results of parameter estimates. $\boldsymbol{A}$, Group-level mean of individual-level posterior mean of $\alpha$ and $\beta$ across moral contexts (good or bad) derived from the winning model. $\boldsymbol{B}$, Scatter plot of individual-level posterior mean of $\alpha$ and $\beta$ across moral contexts (Good or Bad) in each group. Each dot represents the data of a single participant. Error bars represent the SEM; significance: $* * p<0.01$, after controlling for the age difference between groups.

fMRI data preprocessing. Functional imaging data were analyzed using SPM12 (Wellcome Trust Center for Neuroimaging, University College London). The preprocessing procedure followed the pipeline recommended by SPM12. In particular, functional images (EPI) were first realigned to the first volume to correct motion artifacts, unwarped, and corrected for slice timing. Next, the structural T1 image was segmented into white matter, gray matter, and CSF with the skull removed, and coregistered to the mean functional images. Then all functional images were normalized to the Montreal Neurologic Institute (MNI) space, resampled with a $2 \times 2 \times 2 \mathrm{~mm}^{3}$ resolution, in terms of parameters generated in the previous step. Last, the normalized functional images were smoothed using an $8 \mathrm{~mm}$ isotropic full-width at half-maximum based on a Gaussian kernel.

Within-subject representational similarity analyses. To clarify what information rTPJ exactly represents during the decision period that distinguished ASD participants from HC participants, we conducted a within-subject RSA in Python 3.6.8 using the nltools package (version 0.3.14; https://github.com/cosanlab/nltools). Some preparation was performed before implementing RSA. In particular, we established a trialwise general linear model (GLM) for each participant, which included the onsets of the decision screen with the duration of decision time of each valid trial. Here, valid trials were those that conformed to neither the exclusion criterion for the behavioral data (trials with extremely fast or slow responses; see above for details) nor the fMRI data (trials in runs with excessive head motion). The onsets of button press and invalid trials were also modeled as separate regressors of no interest. In addition, six movement parameters were added to this GLM as covariates to account for artifacts of head motion. The canonical hemodynamic response function was used and a high-pass temporal filtering was performed with a default cutoff value of $128 \mathrm{~s}$ to remove lowfrequency drifts. After the parameter estimation, we built up the trial-wise contrasts that were used for subsequent RSA.

Our analyses concentrated on rTPJ given our hypotheses. Notably, we took two different ways to define the cluster of rTPJ to circumvent the potential effect of ROI selection on results. These included defining it via a whole-brain parcellation based on metaanalytic functional coactivation of the Neurosynth database (i.e., the parcellation-based ROI; https://neurovault.org/collections/2099/; including a total of 1750 voxels, with a volume of $2 \times 2 \times 2 \mathrm{~mm}^{3}$ per voxel; same below) or via a coordinate-based manner given a recent meta-analysis on neural correlates of ToM (Schurz et al., 2014; i.e., the coordinate-based ROI; a sphere with a radius of $10 \mathrm{~mm}$ centering on the MNI coordinates of $56 /-56 / 18 ; 515$ voxels in total).

We first extracted the parameter estimates (i.e., contrast value in arbitrary units) of rTPJ from these first-level contrast images of valid trials for each participant, respectively. Next, we constructed the individuallevel neural representation distance matrix (RDM) by computing the pairwise correlation dissimilarity of activation patterns within this mask between each pair of valid trials. We also built up the same neural RDM for left TPJ (ITPJ) as a control region (i.e., the parcellation-based ROI, 1626 voxels in total; the coordinate-based ROI (Schurz et al., 2014), a sphere with a radius of $10 \mathrm{~mm}$ centering on the MNI coordinates of $-53 /-59$ / 20; 515 voxels in total). In line with our research goal, we constructed two main cognitive RDMs in light of the trial-wise information of reputation (i.e., arbitrary code: $0=$ Private, $1=$ Public), and Moral Context (i.e., $0=\mathrm{Bad}, 1=\mathrm{Good}$ ) by calculating the Euclidean distance between each pair of trials. We also built up two additional cognitive RDMs using the trial-wise information of payoffs for the participant (i.e., from 1 to 8 in step of 1), and payoffs for associations (i.e., from 4 to 32 in step of 4) as control subjects. These cognitive RDMs measured the dissimilarity between trials given corresponding information. Notably, we sorted all trials according to the order of Audience, Moral Context, payoff for the participant, and payoff for associations (the charity or the bad cause) to guarantee the information contained by both the neural and cognitive RDMs was matched with each other. To make these cognitive RDMs comparable, we rescaled them within the range from 0 (i.e., the most similar) to 1 (i.e., the most dissimilar). Then we performed a Spearman's rank-order correlation between the neural RDM and the cognitive RDM for each participant.

For the group-level statistical tests, we first implemented the Fisher $r$-to- $z$ transformation on the Spearman's $\rho$, and then performed the permutation-based two-sample $t$ test (i.e., the number of permutations was 5000 ) on these statistics between the two groups for each cognitive RDM separately. To further examine the robustness of these findings, we applied the above analyses using all 256 trials. To this end, a new GLM was established that modeled the onset of the decision screen of all trials to further construct the neural RDM. The remaining details and procedures were the same as mentioned above. 
A

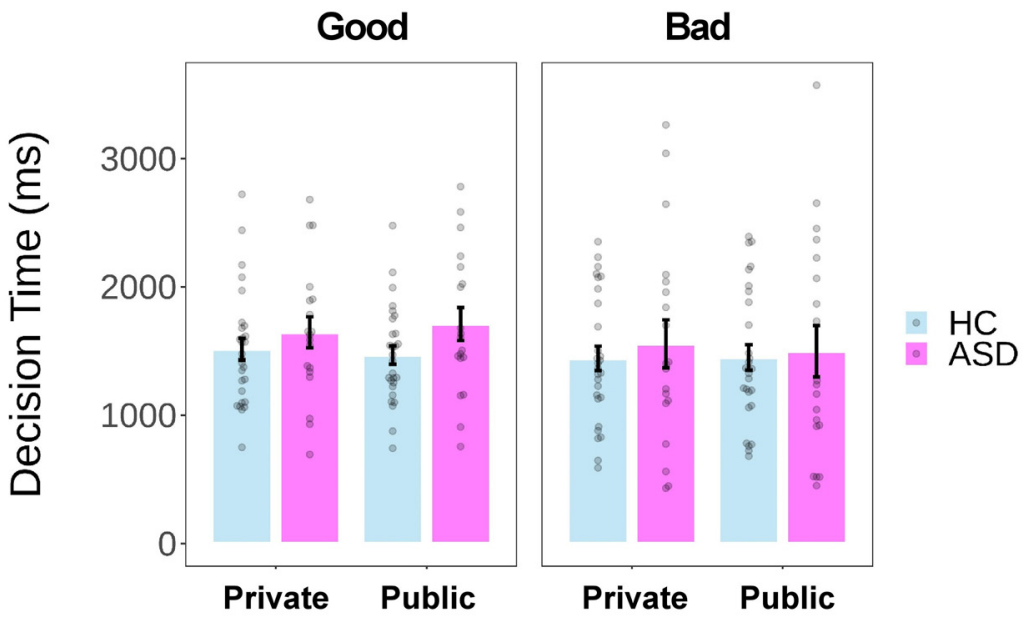

B

HC

Good

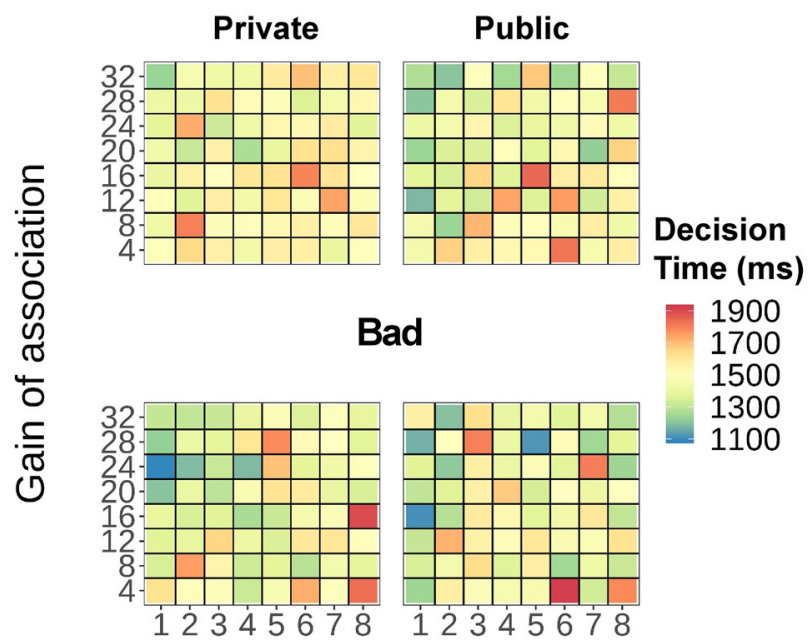

ASD

Good

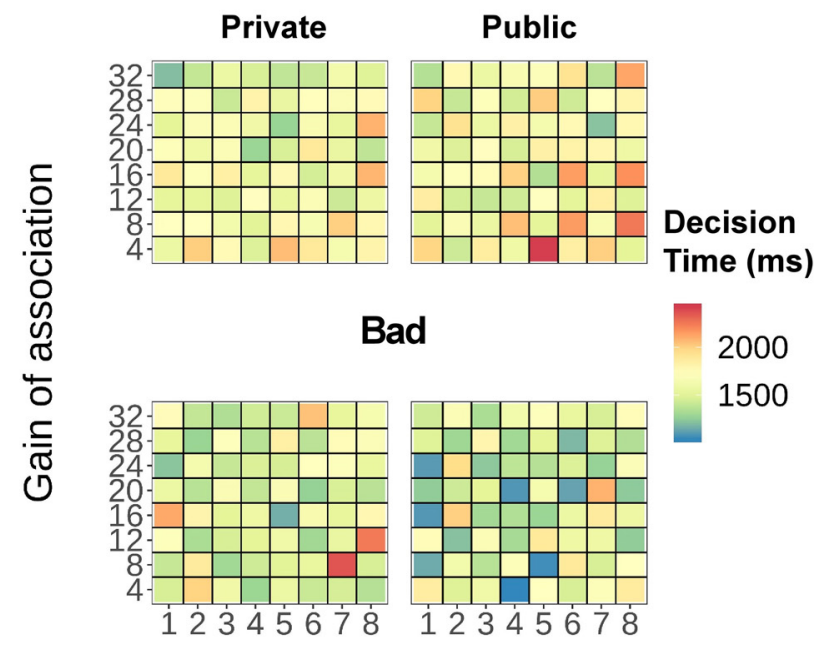

Gain/Loss of participant

Figure 5. Results of decision time (in milliseconds). $\boldsymbol{A}$, Bar plot of the mean decision time as a function of group (ASD or HC), reputation (Private or Public), and context (Good or Bad). $\boldsymbol{B}$, Heat map of the mean decision time regardless of specific choices as a function of payoffs (monetary units) for participants and for associations in each experimental condition of each group.

Supplementary univariate analyses

We also performed a traditional univariate GLM analysis to examine whether the mean neural activations were modulated by different conditions and how neural signals in ASD participants differed from those in healthy control subjects, focusing on the rTPJ. At the individual level, we incorporated the onsets of the decision phase of all conditions (i.e., Private ${ }_{\mathrm{Good}}$, Private $\mathrm{Bad}_{\mathrm{Ba}}$, Public $_{\mathrm{Good}}$, Public $\mathrm{Bad}_{\mathrm{Bad}}$ ) in valid trials as regressors of interest. Similarly, the onsets of button press together with invalid trials as well as head motion parameters were also modeled as separate regressors of no interest. After the parameter estimation, we constructed the following contrasts concerning the main effect of Audience (i.e., Public-Private) and Moral Context (i.e., Good-Bad). These contrast images were fed to the group-level onesample $t$ test for within-group analyses or independent two-sample $t$ tests for between-group analyses. Given the goal of this analysis, we performed a small volume correction within the rTPJ mask. To match the multivariate analyses, we adopted two independent rTPJ masks from different sources (i.e., the parcellation-based ROI and the coordinate-based ROI; see above for details). For the completeness of the analyses, we also performed the same analyses using the ITPJ mask. Otherwise, we adopted a whole-brain threshold of $p<0.001$ uncorrected at the voxel level together with $p<0.05$ FWE corrected at the cluster level (Eklund et al., 2016).

\section{Results}

\section{Subjective evaluation on associations}

Post-task rating on a $0-10$ Likert scale (0 indicates "do not like the association at all," 10 indicates "like the association very much") revealed that both ASD participants and healthy control subjects favored the charity (ASD vs healthy control subjects: $9.3 \pm 1.4$ vs $8.8 \pm 1.2)$ and disliked the bad cause $(0.0 \pm 0.0$ vs $0.3 \pm 0.6)$. No between-group difference was observed in the subjective rating for the charity $\left(b=0.43, \mathrm{SE}=0.39, t_{(44)}=1.11\right.$, $\left.p=0.274, b_{z}=0.17\right)$ and the bad cause $(b=-0.22, \mathrm{SE}=0.14$, $\left.t_{(44)}=-1.56, p=0.125, b_{z}=-0.23\right)$. 
Table 3. Results of mixed-effect logistic regressions predicting log-transformed decision time (in ms)

\begin{tabular}{|c|c|c|c|c|c|}
\hline & $\mathrm{All}^{b}(\mathrm{SE})$ & $\operatorname{Good}^{b}(\mathrm{SE})$ & Good: private ${ }^{b}$ (SE) & Good: public ${ }^{b}$ (SE) & $\operatorname{Bad}^{b}(S E)$ \\
\hline Intercept & $7.22^{* * * * *}(0.07)$ & $7.19^{* * * *}(0.06)$ & $7.19^{\text {***** }}(0.06)$ & $7.18^{* * * *}(0.06)$ & $7.19^{\text {**** }}(0.10)$ \\
\hline Group & $0.07(0.11)$ & $0.04(0.09)$ & $0.04(0.10)$ & $0.09(0.09)$ & $-0.04(0.14)$ \\
\hline Audience & $-0.01(0.02)$ & $-0.02(0.01)$ & & & $0.01(0.01)$ \\
\hline Group $\times$ Audience & $0.04(0.02)$ & $0.04^{*}(0.02)$ & & & $-0.04^{\dagger}(0.02)$ \\
\hline Group $\times$ Moral context & $-0.13^{* * * *}(0.02)$ & & & & \\
\hline Audience $\times$ Moral context & $0.02(0.02)$ & & & & \\
\hline Payoff for oneself ${ }^{\mathrm{a}, \mathrm{b}}$ & & $0.03^{* * * *}(0.01)$ & $0.02^{*}(0.01)$ & $0.04^{* * * *}(0.01)$ & $0.02^{* *}(0.01)$ \\
\hline Payoff for association ${ }^{a, b}$ & & $-0.03^{* * * *}(0.01)$ & $-0.03^{* * *}(0.01)$ & $-0.03^{* * * *}(0.01)$ & $-0.01 *(0.006)$ \\
\hline $\mathrm{Age}^{\mathrm{a}}$ & $0.03(0.05)$ & $-0.001(0.05)$ & $-0.01(0.05)$ & $0.005(0.05)$ & $0.05(0.07)$ \\
\hline AIC & $15,114.8$ & 6095.5 & 3074.3 & 3058.6 & 7203.8 \\
\hline $\mathrm{BIC}$ & $15,203.4$ & 6162.4 & 3122.2 & 3106.5 & 7270.6 \\
\hline$N$ (observation) & 11,823 & 5912 & 2952 & 2960 & 5911 \\
\hline
\end{tabular}

Values are the mean (SE). Reference levels were set as follows: Group, NC; Audience, private; Association, good cause (charity). Table also shows goodness-of-fit statistics: BIC, Bayesian information criterion.

$t p<0.06, * p<0.05, * * p<0.01, * * * p<0.001$

${ }^{a}$ We standardized these variables for the analyses.

${ }^{b}$ These variables were added as covariates only when the regressor Association (and its interaction) was not in the regression model, as the regressor "payoff for oneself" qualitatively covaried with Association, which might cause the collinear issue.

ASD participants do not appear to consider social reputation and rigorously conform to a rule in curbing their immoral behaviors

Mixed-effect logistic regressions revealed that participants were more likely to behave morally in the Bad Context than in the Good Context (i.e., rejecting more frequently the offer in the Bad Context than accepting it in the Good Context; a main effect of Moral Context: $\left.\chi^{2}(1)=632.68, p<0.001\right)$. More importantly, significant interaction effects were identified between Group and Audience $\left(\chi^{2}(1)=4.50, p=0.034\right)$ as well as between Group and Moral Context $\left(\chi^{2}(1)=59.33, p<0.001\right)$ on choosing the moral option (i.e., accepting the offer to benefit a charity or rejecting the offer to benefit a morally bad cause; Fig. 2). No other main effect $(p$ values $>0.09)$ or interaction effect was detected ( $p$ values $>0.57)$.

To understand the first interaction effect, we performed post hoc analyses on the dataset of the ASD and the HC groups, respectively. For each analysis, we ran a similar logistic regression, including the main effect of audience and context as the fixed-effects predictors. The Audience $\times$ Moral Context interaction was dropped from these analyses as neither this effect $\left(\chi^{2}(1)=0.31, p=0.580\right)$ nor the three-way interaction effect $\left(\chi^{2}(1)=0.30, p=0.586\right)$ was significant in the main analysis. The results showed that while healthy control subjects were more likely to make the moral choice when they were observed in the Public condition (vs Private; $\mathrm{OR}=1.16, b=0.15, \mathrm{SE}=0.06$, $p=0.012$ ), ASD participants did not change their behaviors significantly depending on the presence or absence of a witness $(\mathrm{OR}=0.93, b=-0.08, \mathrm{SE}=0.08, p=0.371)$.

To understand the second interaction effect, we performed similar regression analyses using trials in the Good and Bad Contexts separately. For each post hoc regression analysis, we incorporated Group and Audience, along with their interaction as the fixed-effects predictors, while controlling for the effect of the payoff for participants and associations in these analyses (same below for analyses on decision time). We observed only a strong main effect of Group $\left(\chi^{2}(1)=5.05, p=0.025\right)$ and a Group $\times$ Audience interaction effect in the Bad Context $\left(\chi^{2}(1)=4.04, p=0.044\right)$, which was mainly driven by a drastically enhanced probability of behaving morally in the ASD group (vs
$\mathrm{HC}$ group) when deciding privately $(\mathrm{OR}=64.25, b=4.16, \mathrm{SE}=$ $1.53, p=0.006)$. Neither of these effects was significant in the Good Context ( $p$ values $>0.12$; Table 2, details of regression outputs).

\section{ASD participants evaluate the immoral gains more severely for both themselves and the bad cause}

We developed eight models with different utility functions characterizing participants' choices in the ASD and HC groups separately. Model estimation and comparison was performed with an HBA approach (Gelman et al., 2014) via the "hBayesDM" package (see Materials and Methods for details). R-hat values of all estimated parameters of all models are close to 1.0 (i.e., $<1.06$ in the worst case), which showed sufficient convergence of the MCMC chains (Gelman and Rubin, 1992). Hierarchical Bayesian model comparison showed that model 4 (see below for the utility function) has the lowest LOOIC scores, indicating that it fits to the current dataset the best compared with other competitive models (Fig. 3A), as follows:

$$
\begin{gathered}
\operatorname{SV}\left(M_{S}, M_{O}\right) \\
=\left\{\begin{array}{c}
-\left(\alpha_{\mathrm{Good}}-q * \theta\right) * M_{S}+\left(\beta_{\mathrm{Good}}+q * \theta\right) * M_{O} \text { if Good } \\
\left(\alpha_{\mathrm{Bad}}-q * \theta\right) * M_{S}+\left(\beta_{\mathrm{Bad}}-q * \theta\right) * M_{O} \text { if } \mathrm{Bad}
\end{array}\right.
\end{gathered}
$$

Here, SV denotes the subjective value of the given trial depending on the specific choice made by the participant. $M_{s}$ and $M_{o}$ represent the payoff (gain or loss) for oneself and each association respectively. Established on the basis of a donation task (Lopez-Persem et al., 2017), the winning model assumed that people weighed their own payoff (measured by $\alpha: \alpha_{\text {good, }}$, $\alpha_{\text {bad }}$ ) and the benefits for associations (measured by $\beta: \beta_{\text {good, }}$ $\beta_{\text {bad }}$ ) separately given the moral contexts involved in the decisions. $\theta$ measured the audience effect, which was modulated by an indicator function, $q$ ( 0 for private, 1 for public; see Materials and Methods for details). A posterior predictive check further confirmed that the simulated choice behaviors in light of the parameter estimates of the winning model can nicely capture the actual behaviors by showing a high correlation between each 


\section{Multivoxel Patterns}
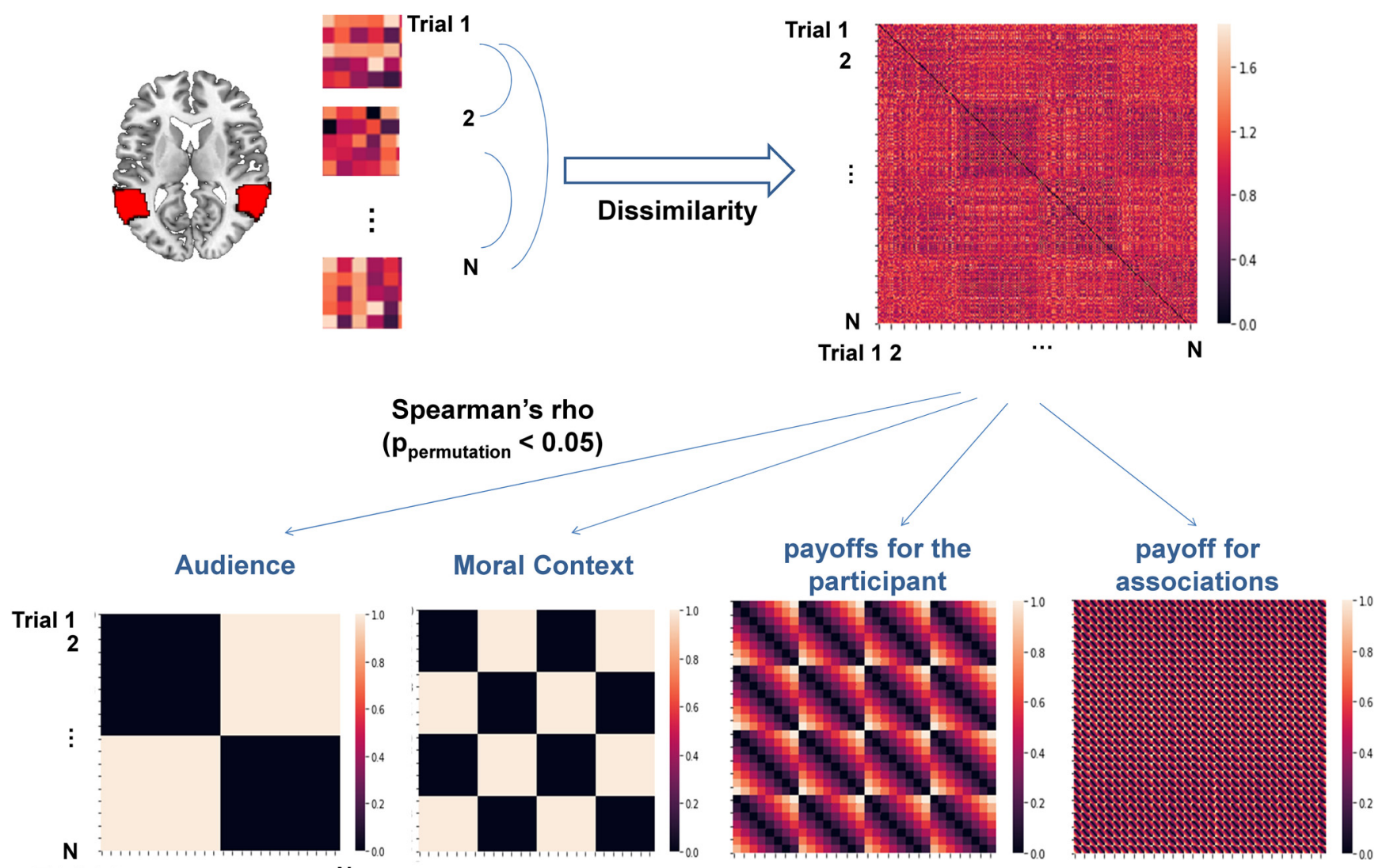

Trial 12

$\cdots$

$\mathbf{N}$

\section{Cognitive RDM}

Figure 6. Illustration of within-subject RSAs. For each individual, we first constructed a neural RDM measuring the correlational distances of multivoxel patterns of the decision-relevant neural activities within either left or right TPJ between each pair of valid trials, respectively. Next, we constructed four cognitive RDMs by calculating the Euclidean distances between each pair of valid trials with respect to the following information: (1) Audience (i.e., social reputation; Private or Public); (2) Moral Context (i.e., Good or Bad); (3) payoffs for the participant; and (4) payoffs for associations. Notably, we sorted all trials according to the order of Audience, Moral Context, payoff for the participant, and payoff for associations to guarantee the information contained by both the neural and cognitive RDMs was matched with each other. Then we performed the Spearman rank-ordered correlation between the neural and the cognitive RDMs. Finally, an independent two-sample permutation-based $t$ test was conducted to compare the between-group difference on the $z$-transformed Spearman's $\rho$.

other (i.e., for both the HC group and ASD group: Pearson's $r$ values $>0.99, p$ values $<0.001$; Fig. $3 B)$.

Next, we examined how parameters derived from the winning model vary in terms of groups and experimental conditions. To this end, we extracted the individual-level posterior mean of key parameters (i.e., $\alpha, \beta$, and $\theta$ ) and performed linear regression, including Group as the predictor on each of them, respectively. To test the Group $\times$ Association interaction on $\alpha$ and $\beta$, we regressed groups on the difference score between two contexts for each of the parameters. For all these regression analyses, we also added age as a covariate to control for its confounding effect.

We first showed a significant Group $\times$ Association interaction on both $\alpha\left(b=7.93, \mathrm{SE}=3.91, t_{(44)}=2.03, p=0.049 ; b_{z}=\right.$ $0.30)$ and $\beta\left(b=-10.88, \mathrm{SE}=3.46, t_{(44)}=-3.14, p=0.003 ; b_{z}=\right.$ $-0.43)$. Simple-effect analyses showed a significant decrease of decision weights on payoffs, in ASD participants, for both themselves $\left(\alpha_{\mathrm{HC}}\right.$ vs $\alpha_{\mathrm{ASD}}: 0.90 \pm 11.74$ vs $-9.27 \pm 10.13, t_{(44)}=$ $\left.-3.14, p=0.003 ; b_{z}=-0.45\right)$ and the morally bad cause ( $\beta_{\mathrm{HC}}$ vs $\beta_{\mathrm{ASD}}:-4.87 \pm 5.02$ vs $-11.52 \pm 8.67, t_{(44)}=-2.96, p=0.005 ; b_{z}$ $=-0.41)$. No between-group difference was observed in either parameter when participants weighed the tradeoff between personal financial losses ( $\alpha_{\mathrm{HC}}$ vs $\alpha_{\mathrm{ASD}}$ : $11.18 \pm 6.41$ vs $8.89 \pm 9.09$, $\left.t_{(44)}=-1.26, p=0.216 ; b_{z}=-0.19\right)$ and the donation to a charity
( $\beta_{\mathrm{HC}}$ vs $\beta_{\mathrm{ASD}}: 4.38 \pm 4.04$ vs $6.78 \pm 7.83, t_{(44)}=1.56, p=0.126$; $b_{z}=0.24$; Fig. $4 A$ ). Notably, the correlation between $\alpha$ and $\beta$ was not significant across moral contexts in either group (ASD group: Good Context: $r=-0.177, p=0.469$; Bad Context: $r=$ $-0.242, p=0.319$; HC group: Good Context: $r=-0.018$, $p=0.928$; Bad Context: $r=-0.003, p=0.989$; Fig. 4B). This indicates that participants value payoffs for oneself and the causes (associations) independently. Consistent with the behavioral finding, we also observed a trend to significance for the betweengroup difference in $\theta$; namely, that ASD participants exhibited a reduced audience effect compared with HC participants during moral decision-making ( $\theta_{\mathrm{HC}}$ vs $\theta_{\mathrm{ASD}}: 0.39 \pm 0.67$ vs $0.17 \pm 0.12$, $\left.t_{(44)}=-1.80, p=0.080, b_{z}=-0.27\right)$.

ASD participants do not differ from HCs in decision time in either moral context

Mixed-effect linear regression on log-transformed decision time showed a significant three-way interaction among Group, Audience, and Moral Context $\left(F_{(1,11,769)}=6.02, p=0.014\right)$, along with a Group $\times$ Moral Context interaction effect $\left(F_{(1,11,769)}=\right.$ 100.20, $p<0.001)$ and a main effect of Moral Context $\left(F_{(1,11,772)}=299.76, p<0.001\right)$ after controlling for the effect of specific choices $\left(F_{(1,11,804)}=3.76, p=0.052\right.$; Fig. 5). Splitting the 
A

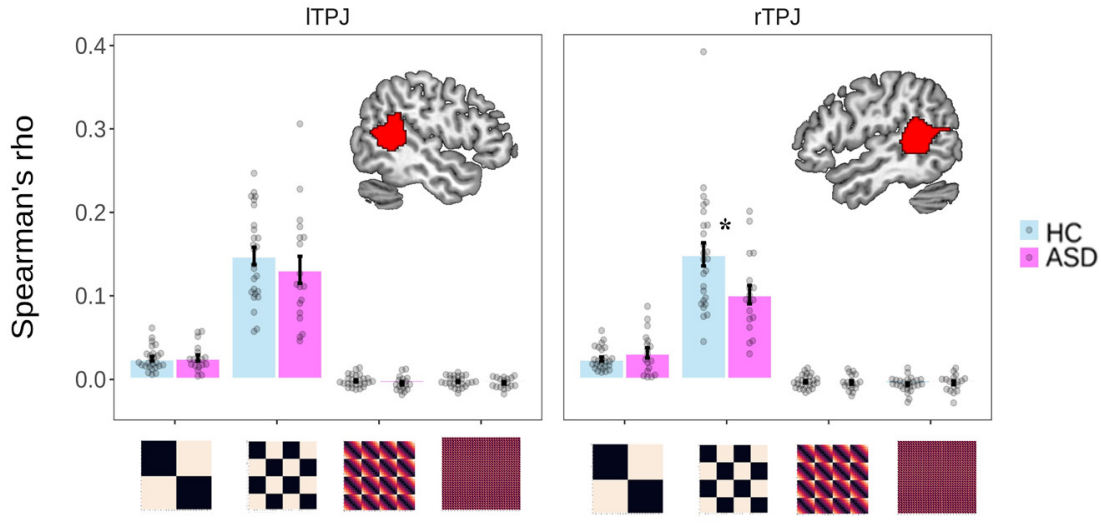

B

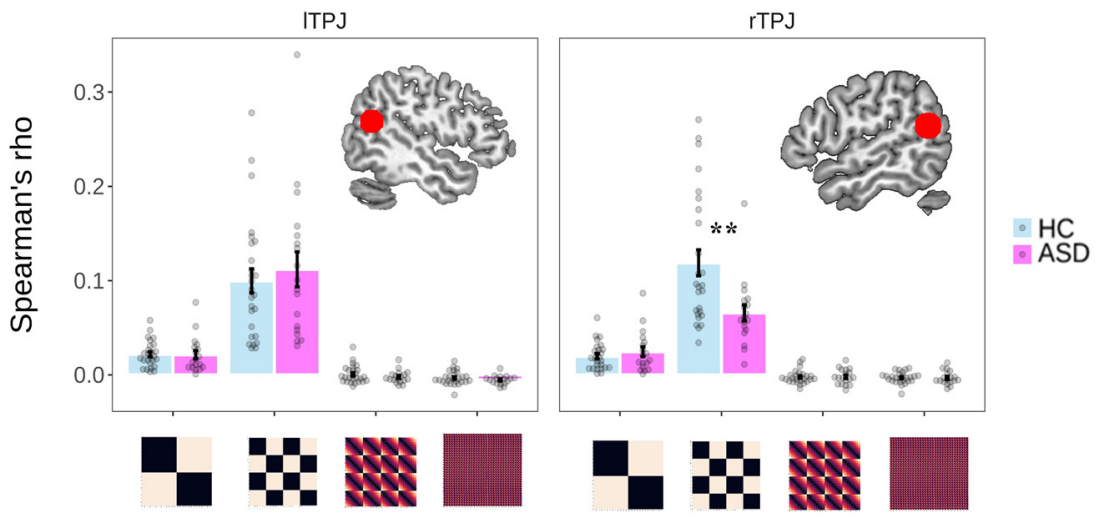

Figure 7. $\quad \boldsymbol{A}, \boldsymbol{B}$, Within-subject RSA results using the parcellation-based ROI $(\boldsymbol{A})$ and the coordinate-based ROI $(\boldsymbol{B})$ of TPJ. For each participant, we only adopted valid trials (see Materials and Methods for details) in these analyses. Each dot represents the data of a single participant. Error bars represent the SEM; significance: $* p_{\text {permutation }}<0.05, * * p_{\text {permutation }}<0.01$, after controlling for the age difference.

dataset according to Moral Context, post hoc analyses revealed a significant Group $\times$ Audience interaction effect when participants decided whether to serve a good cause at a personal cost $\left(F_{(1,5860)}=4.28, p=0.039\right)$ and a trend-to-significant interaction effect in the Bad Context $\left(F_{(1,5859)}=3.76, p=0.053\right)$. However, neither the main effect of Group in the Good Context (ASD: $1676.5 \pm 527.7 \mathrm{~ms}$; HC: $1490.0 \pm 399.5 \mathrm{~ms} ; F_{(1,44)}=0.51, p=$ 0.479 ) nor in the Bad Context (ASD: $1525.7 \pm 828.1 \mathrm{~ms}$; HC: $\left.1445.5 \pm 500.9 \mathrm{~ms} ; F_{(1,44)}=0.17, p=0.682\right)$ was significant. The interaction effect in the Good Context was driven by a slightly larger difference in decision time between groups when they made decisions in public (ASD: $1709.5 \pm 558.8 \mathrm{~ms}$; HC: $1467.9 \pm$ $379.3 \mathrm{~ms}$ ) compared with those made in private (ASD: $1645.5 \pm$ $526.0 \mathrm{~ms}$; HC: $1514.1 \pm 448.9 \mathrm{~ms}$ ). However, neither of these between-group differences was statistically significant (public: $b=0.09, \quad \mathrm{SE}=0.09, t_{(44)}=0.98, \quad p=0.334, b_{z}=0.29$; private: $b=0.04, \mathrm{SE}=0.10, t_{(44)}=0.42, p=0.678, b_{z}=0.13$; Table 3, details of regression output).

\section{Imaging results}

Decreased neural representation of moral contexts in the rTPJ of ASD participants

To examine how the decision-related neural patterns differ in representing information contributing to the value computation and final decisions between ASD participants and $\mathrm{HC}$ participants, we performed a withinsubject RSA (Fig. 6, illustration of RSA procedure). Given our hypotheses, we focused our analysis on the rTPJ. To avoid bias on results caused by ROI selection to the maximum degree, we defined the rTPJ in two different ways, either via a whole-brain parcellation based on meta-analytic functional coactivation of the Neurosynth database (i.e., the parcellation-based ROI) or via a coordinate-based manner given a recent meta-analysis on neural correlates of ToM (Schurz et al., 2014; i.e., the coordinate-based ROI; for details, see Materials and Methods).

Regardless of the ROI approach, we consistently found that, compared with the HC group, ASD participants only showed a reduced representation of the information of the identity of associations in the rTPJ (ASD vs HC: the parcellation-based ROI: Spearman's $\rho=0.101 \pm 0.047$ vs 0.150 $\pm 0.071 ; p_{\text {permutation }}=0.013$; the coordinate-based ROI: $0.066 \pm 0.036$ vs $0.119 \pm 0.070 ; p_{\text {permutation }}=$ $0.006)$. These significant differences held after ruling out the confounding effect of age. Importantly, such a between-group difference of similarity was not observed between the neural $\mathrm{RDM}$ in the rTPJ and other cognitive RDMs (the parcellation-based ROI: $p_{\text {permutation values }}>0.20$; the coordinate-based ROI: $p_{\text {permutation }}$ values $>$ 0.38 ) or between the neural RDM in the ITPJ and all the cognitive RDMs (the parcellation-based ROI: $p_{\text {permutation }}$ values $>0.17$; the coordi-

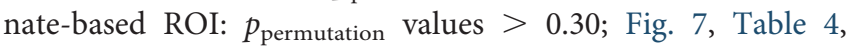
details). Post hoc 2 (group) $\times 4$ (cognitive RDM) mixed ANOVA on the Fisher $r$-to- $z$ transformed Spearman's $\rho$ revealed a strong interaction between group and cognitive RDM only in rTPJ (the parcellation-based ROI: $F_{(3,126)}=6.09, p<0.001$; the coordinate-based ROI: $\left.F_{(3,126)}=8.37, p<0.001\right)$ but not in ITPJ (the parcellation-based ROI: $F_{(3,126)}=0.65, p=0.585$; the coordinate-based ROI: $\left.F_{(3,126)}=0.42, p=0.743\right)$ after controlling for the age difference, which further confirmed that the reduced ability to represent the information of moral context in ASD participants was uniquely reflected in rTPJ. Finally, to further examine the robustness of the above findings, we also applied the above analyses using all 256 trials, which did not affect the results (Fig. 8, Table 5, details).

\section{Univariate results in $r T P J$}

We first investigated whether the neural audience effect in rTPJ (i.e., Public $>$ Private) in healthy control subjects reported in the study by Qu et al. (2019) could be replicated in the present study. The results showed that the rTPJ activity was not significantly higher in the Public (vs Private) condition (no voxel survived under a threshold of $p<0.005$ uncorrected at the voxel level 
Table 4. Within-subject RSA results in TPJ using valid trials

\begin{tabular}{|c|c|c|c|c|c|c|}
\hline & & & \multicolumn{2}{|c|}{ Spearman's $\rho$ (mean \pm SD) } & \multirow[b]{2}{*}{$p_{\text {permutation }}$} & \multirow[b]{2}{*}{$p_{\text {permutation }}{ }^{\mathrm{b}}$} \\
\hline & & & ASD & $H C$ & & \\
\hline \multirow[t]{6}{*}{ Neurosynth } & ITPJ & Audience & $0.026 \pm 0.016^{* * * *}$ & $0.025 \pm 0.014^{* * * *}$ & 0.848 & 0.493 \\
\hline & & Payoff for oneself & $-0.005 \pm 0.008$ & $-0.002 \pm 0.007$ & 0.174 & 0.271 \\
\hline & & Payoff for association & $-0.004 \pm 0.006$ & $-0.003 \pm 0.006$ & 0.413 & 0.447 \\
\hline & rTPJ & Audience & $0.032 \pm 0.025^{* * * *}$ & $0.024 \pm 0.013^{* * * *}$ & 0.201 & 0.163 \\
\hline & & Moral context & $0.101 \pm 0.047^{* * * *}$ & $0.150 \pm 0.071^{\text {***** }}$ & 0.013 & 0.018 \\
\hline & & Payoff for association & $-0.004 \pm 0.010$ & $-0.006 \pm 0.009$ & 0.578 & 0.995 \\
\hline \multirow[t]{5}{*}{ Meta-analysis $^{\mathrm{a}}$} & ITPJ & Audience & $0.021 \pm 0.019^{* * * *}$ & $0.022 \pm 0.014^{* * * *}$ & 0.912 & 0.931 \\
\hline & & Moral context & $0.112 \pm 0.079^{* * * *}$ & $0.100 \pm 0.065^{\text {**** }}$ & 0.566 & 0.551 \\
\hline & & Payoff for oneself & $-0.002 \pm 0.007$ & $0.0005 \pm 0.009$ & 0.304 & 0.472 \\
\hline & & Payoff for association & $-0.005 \pm 0.005$ & $-0.003 \pm 0.007$ & 0.308 & 0.262 \\
\hline & rTPJ & Audience & $0.025 \pm 0.022^{* * * *}$ & $0.020 \pm 0.014^{* * * *}$ & 0.383 & 0.230 \\
\hline
\end{tabular}

We excluded trials that did not reach the behavioral criterion (i.e., those with a decision time of $<200 \mathrm{~ms}$ or longer than the mean \pm 3 SDs of that individual) or fMRI criterion (all trials in a run with an excessive head motion: ASD, >5 mm; HC, >3 mm). I, Left; r, right.

${ }^{\text {a }}$ These masks were spheres with a radius of $10 \mathrm{~mm}$ centering on the MNI coordinates based on a recent meta-analysis involving the mentalizing process (peak MNI coordinates: left TPJ/pSTS: $-53 /-59 / 20$; right TPJ/pSTS: $56 /-56 / 18)$.

${ }^{\mathrm{b}}$ We added the standardized age as the covariates to the regression, using the ImPerm package.

**** These effects are significantly higher than 0 (i.e., one-sample $t$ test with 5000 permutations; $p_{\text {permutation }}<0.001$ ).

with $k=10$, in either rTPJ mask; Fig. $9 A)$. One possibility could be that the neural audience effect of rTPJ was modulated by large individual differences in the behavioral audience effect across individuals, which blurred the main effect. To test this possibility, we extracted the mean activity (contrast value) of the rTPJ from each condition, and then computed a neural index of audience effect for each individual (i.e., $0.5 *\left[\right.$ (Public $_{\mathrm{Good}}+$ Public $\left._{\mathrm{Bad}}\right)$ - $\left(\right.$ Private $_{\mathrm{Good}}+$ Private $\left.\left.\left._{\mathrm{Bad}}\right)\right]\right)$. We also defined a behavioral index of audience effect on the proportion of moral choice, which was calculated with the same equation. Results showed that the Pearson correlation between these two indices was not significant (the parcellation-based ROI: $r(24)=0.02, p=0.914$; the coordinate-based ROI: $r(24)=$ $-0.06, p=0.761$; Fig. $9 B$ ). Furthermore, the between-group comparison did not reveal a significant result in the audience effect in rTPJ (i.e., no voxel survived under the threshold mentioned above; Fig. 10). Besides, no significant difference in the neural activity was observed in the rTPJ between the Good and Bad Contexts in the HC group or between two groups (i.e., no voxel survived under the threshold mentioned above). For the completeness of the analyses, we also applied the same analyses to ITPJ, yielding similar results (Figs. 9, 10, Table 6, whole-brain results under a liberal threshold).

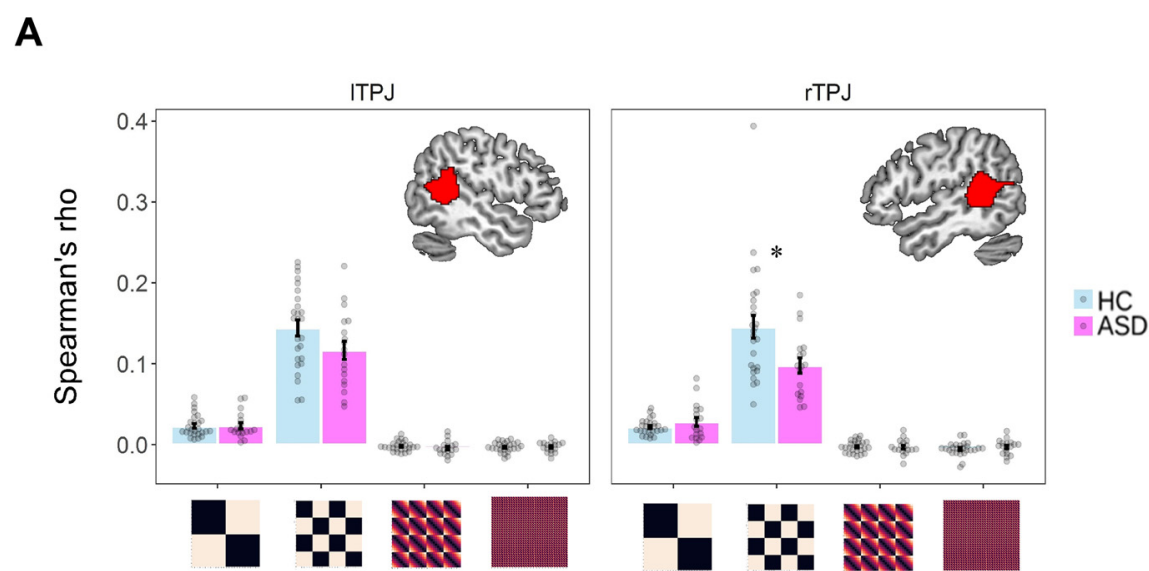

B

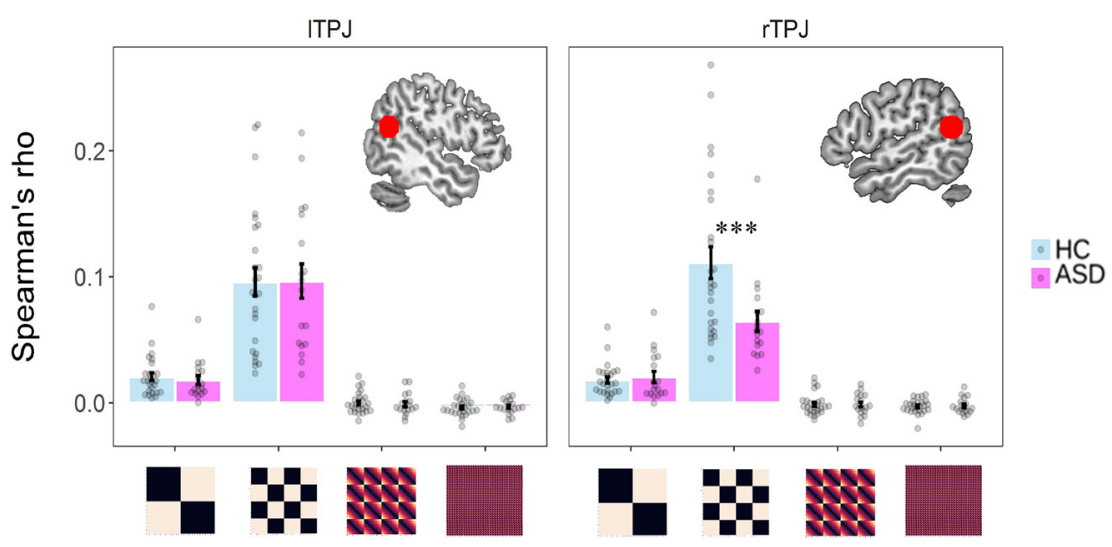

Figure 8. $A, B$, Robustness check of within-subject RSA results using the parcellation-based ROI $(A)$ and the coordinate-based ROI (B) of TPJ. For each participant, we adopted all 256 trials in these analyses. Each dot represents the data of a single participant. Error bars represent the SEM; significance: $* p_{\text {permutation }}<0.05, * * * p_{\text {permutation }}<0.001$, after controlling for the age difference. 
Table 5. Within-subject RSA results in TPJ using all 256 trials

\begin{tabular}{|c|c|c|c|c|c|c|}
\hline & & & \multicolumn{2}{|c|}{ Spearman's $\rho$ (mean \pm SD) } & \multirow[b]{2}{*}{$p_{\text {permutation }}$} & \multirow[b]{2}{*}{$p_{\text {permutation }}{ }^{\mathrm{b}}$} \\
\hline & & & ASD & HC & & \\
\hline \multirow[t]{5}{*}{ Neurosynth } & ITPJ & Audience & $0.023 \pm 0.016^{* * * *}$ & $0.023 \pm 0.014^{* * * *}$ & 0.967 & 0.538 \\
\hline & & Payoff for oneself & $-0.004 \pm 0.009$ & $-0.002 \pm 0.006$ & 0.299 & 0.358 \\
\hline & & Payoff for association & $-0.003 \pm 0.006$ & $-0.003 \pm 0.006$ & 0.932 & 0.919 \\
\hline & rTPJ & Audience & $0.028 \pm 0.022^{* * * *}$ & $0.022 \pm 0.010^{* * *}$ & 0.203 & 0.169 \\
\hline & & Payoff for association & $-0.003 \pm 0.009$ & $-0.005 \pm 0.008$ & 0.386 & 0.667 \\
\hline \multirow[t]{5}{*}{ Meta-analysis $^{\mathrm{a}}$} & ITPJ & Audience & $0.018 \pm 0.015^{* * * *}$ & $0.021 \pm 0.016^{* * * *}$ & 0.579 & 0.850 \\
\hline & & Moral context & $0.097 \pm 0.058^{* * * *}$ & $0.096 \pm 0.058^{* * * *}$ & 0.972 & 0.914 \\
\hline & & Payoff for oneself & $-0.001 \pm 0.008$ & $-0.00004 \pm 0.008$ & 0.721 & 0.745 \\
\hline & & Payoff for association & $-0.003 \pm 0.005$ & $-0.004 \pm 0.007$ & 0.775 & 0.871 \\
\hline & rTPJ & Audience & $0.021 \pm 0.019^{* * * *}$ & $0.018 \pm 0.013^{* * *}$ & 0.613 & 0.451 \\
\hline
\end{tabular}

I, left; $r$, right. Post hoc 2 (group) $\times 4$ (cognitive RDM) mixed ANOVA on the Fisher $r$-to-z transformed Spearman's $\rho$ revealed a strong interaction between group and cognitive RDM only in rTPJ regardless of the way we defined the ROI (the parcellation-based ROI: $F_{(3,126)}=6.59, p<0.001$; the coordinate-based R0I: $F_{(3,126)}=7.37, p<0.001$ ), which was not true in ITPJ (the parcellation-based Rol: $F_{(3,126)}=3.00, p=0.033$; the coordinatebased ROI: $\left.F_{(3,126)}=0.03, p=0.994\right)$ after controlling for the age difference, which further confirmed that the specific between-group effect in representing information of Moral context was unique in rTPJ.

${ }^{a}$ These masks were spheres with a radius of $10 \mathrm{~mm}$ centering on the MNI coordinates based on a recent meta-analysis involving the mentalizing process (peak MNI coordinates: left TPJ/pSTS: - 53/-59/20; right TPJ/pSTS: $56 /-56 / 18)$.

${ }^{\mathrm{b}}$ We added the standardized age as the covariates to the regression, using the ImPerm package.

**** These effects are significantly higher than 0 (i.e., one-sample $t$ test with 5000 permutations; $p_{\text {permutation }}<0.001$ ).

\section{Discussion}

When facing moral dilemmas such as earning ill gotten money by supporting a bad cause or donating to a charity at a personal cost, how do autistic individuals choose? Do they vary their immoral/moral behaviors with respect to the presence or absence of someone else or contingent on moral concerns elicited by specific contexts (i.e., serving a good or a bad cause)? What neurocomputational mechanisms underlie such behavioral changes? In the present model-based fMRI study, we attempted to answer these questions by adopting a novel task in which individuals decided among tradeoffs between personal benefits/losses and context-sensitive moral concerns while also, perhaps, considering their social reputation. Our behavioral results reveal that the moral behavior of ASD individuals differs from healthy control subjects in two aspects.

First, ASD individuals, unlike healthy control subjects, blurred the distinction between private and public conditions while making moral decisions. This finding not only coheres with the ToM deficit hypothesis of ASD individuals (BaronCohen et al., 1985; Baron-Cohen, 2001), but also agrees with previous findings using a tradeoff between suffering personal losses and donating to a good cause (Izuma et al., 2011). Moreover, it extends the lack of attention to social reputation in autism to include an immoral context where individuals are confronted with a moral conflict between personal profits and a cost brought by benefiting an immoral cause. This first finding confirms that ASD individuals do not appear to take into account their social reputation while making immoral/moral choices consistently across contexts (Izuma et al., 2011).

Second, a robust behavioral difference between ASD individuals and healthy control subjects was found specifically in one moral context. ASD individuals generally refused more offers in the Bad Context that could have earned extra money for themselves but resulted in an immoral consequence. No similar between-group difference was observed in the Good Context. Note that decision difficulty cannot explain these behavioral effects because no decision time difference was observed between the two groups. Furthermore, this effect cannot be attributed to their greater dislike/like for the morally bad cause because there was no significant between-group difference on subjective ratings.

Our computational modeling approach provides crucial insights to understand further this difference in ASD individuals, which is specific to moral behaviors serving a bad cause. In parallel to the choice findings, ASD individuals drastically lowered their decision weights on payoffs that would be earned both for themselves and the morally bad cause, whereas they valued the personal losses and the benefits of the charity similarly to healthy control subjects. These findings strongly indicate an atypical valuation of morally tainted personal profits and moral costs brought by benefiting a bad cause in autistic individuals. This probably led to their extremely high rejection rate for immoral offers. Our results fit the literature on moral judgment, which has shown that ASD individuals exhibit an excessive valuation of negative consequences when judging the moral appropriateness or permissibility of actions. For example, Moran et al. (2011) reported that ASD participants considered accidental negative outcomes less permissible than healthy control subjects, whereas both groups rated other types of events as having similar moral appropriateness. In a more recent study, a similar effect was observed; namely, ASD individuals judged a protagonist's immoral but understandable action (e.g., a husband stealing medicine sold at an unaffordable price to save his fatally sick wife) as less morally acceptable than did healthy control subjects (Schaller et al., 2019). In agreement with these findings, our results suggest that autistic individuals may apply a rule of refusing to serve an immoral cause because they evaluate the negative consequences of their actions more severely. This might result in insensitivity in ASD individuals who have difficulty in adjusting their behaviors regarding their personal interests that might be associated with immoral consequences.

Another possible explanatory factor of ASD participants' tendency to make more moral decisions in the Bad Context is behavioral rigidity, a core symptom for clinical diagnosis of ASD (American Psychiatric Association, 2013). Previous studies have revealed that, compared with healthy control subjects, individuals with ASD were more likely to show repetitive behaviors in a 
variety of cognitive tasks (D'Cruz et al., 2013; Watanabe et al., 2019). Hence, it is possible that behavioral rigidity, at least to some extent, is a more general mechanism that contributes to the inflexibly moral behaviors in the Bad Context (i.e., rejecting $>85 \%$ of the trials). Nonetheless, this explanation should be treated with caution because it seems not to account well for the behaviors of ASD participants in the Good Context, where they behaved in a comparatively more flexible fashion (i. e., accepting $\sim 60 \%$ of the trials).

At the brain level, we performed within-subject RSA to examine how different types of information (social reputation, moral contexts, payoffs for each party) that contribute to the final decision were represented in the rTPJ, and how distinct rTPJ representations distinguish ASD participants from healthy control subjects. Compared with the traditional univariate approach, RSA takes advantage of neural patterns from multiple voxels and proves to be more sensitive to subtle experimental effects that might be masked by the averaged local neural responses (Norman et al., 2006; Hebart and Baker, 2018). RSA is also considered to be more informative, because it takes into account the variability within multivoxel patterns (Kriegeskorte et al., 2008; Popal et al., 2019). We observed a reduced association (representation similarity) in ASD participants (vs healthy control subjects) between the trial-by-trial multivariate rTPJ patterns and the information structure unique to the moral contexts, despite that, such a representation in rTPJ is present in both groups. The representations of other types of information (i.e., social reputation and payoffs for each party) did not differ between groups. Together with a much higher rejection rate, as well as atypical weights on payoffs in the bad context, this RSA finding provides a neural account for previous findings that autistic individuals are inclined to judge moral culpability more severely than HCs on the basis of its consequences. This distinguishes ASD individuals from HCs, who prioritize intentions to guide their moral judgments (Fadda et al., 2016; Salvano-Pardieu et al., 2016; Bellesi et al., 2018). Notably, our results showed that the group difference in representational similarity was only detected in rTPJ but not in ITPJ, further indicating a unique role of rTPJ in specifically representing information concerning moral contexts.

Regarding the function of the rTPJ, our RSA finding is consistent with a recent TMS study in healthy volunteers that revealed a context-sensitive moral role of rTPJ in signaling moral conflicts between personal benefits and moral values (Obeso et al., 2018). That study evidenced an asymmetrical TMS effect of rTPJ on moral behaviors depending on the moral context.

B

\section{Parcellation-Based ROI}

ITPJ

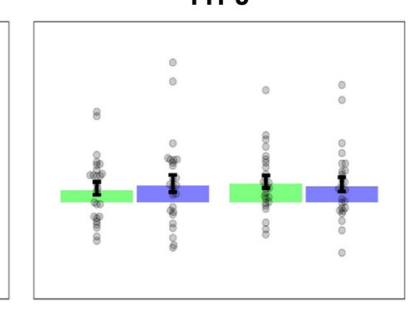

Coordinate-Based ROI

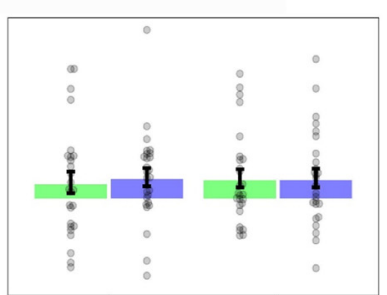

Private

Public

Public

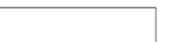

Private

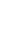

Private

\begin{abstract}
Public
\end{abstract}

\section{Parcellation-Based ROI}
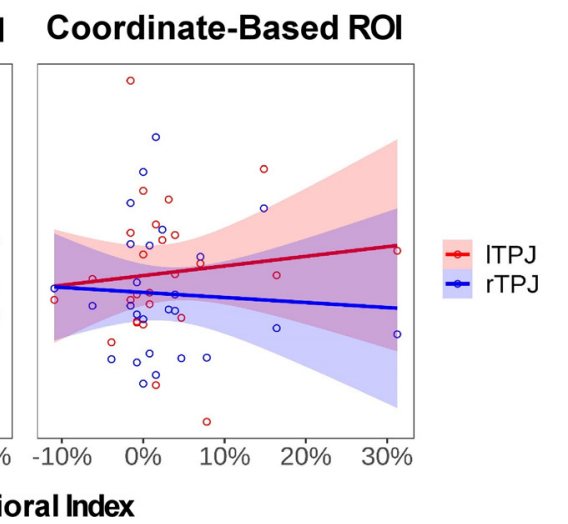

\section{Behavioral Index}

Figure 9. Univariate results of TPJ in healthy control subjects. $A$, Bar plot of TPJ signals. For visualization, we extracted the mean activity (contrast value) of ITPJ and rTPJ from the parcellation-based or coordinate-based mask as a function of reputation (Private or Public) and context (Good or Bad). Each dot represents the data of a single participant. Error bars represent the SEM. $\boldsymbol{B}$, Relationship between neural audience effect in TPJ and behavioral audience effect across individuals. Each dot represents the data of a single participant. Each line represents the linear fit. Shaded areas represent the $95 \%$ confidence interval.

Specifically, healthy participants under rTPJ stimulation were more altruistic such that they accepted more offers of donating to a charity at a personal cost regardless of donation amounts, whereas rTPJ disruption inhibited participants from accepting offers to earn morally tainted money only when benefits to the bad cause were large. Building on this finding, the present study provides further evidence using a different approach to reveal that rTPJ is critically involved in representing the moral contexts that flexibly modulate the tradeoff between personal benefits and other's welfare during decision-making, which extends our understanding of the rTPJ function.

Notably, our univariate fMRI results did not reveal a neural audience effect in rTPJ in the healthy control subjects as was initially expected. Although previous studies provided evidence (Izuma, 2012; Qu et al., 2019) suggesting that TPJ is involved in social reputation, negative evidence also exists. For instance, a 
A

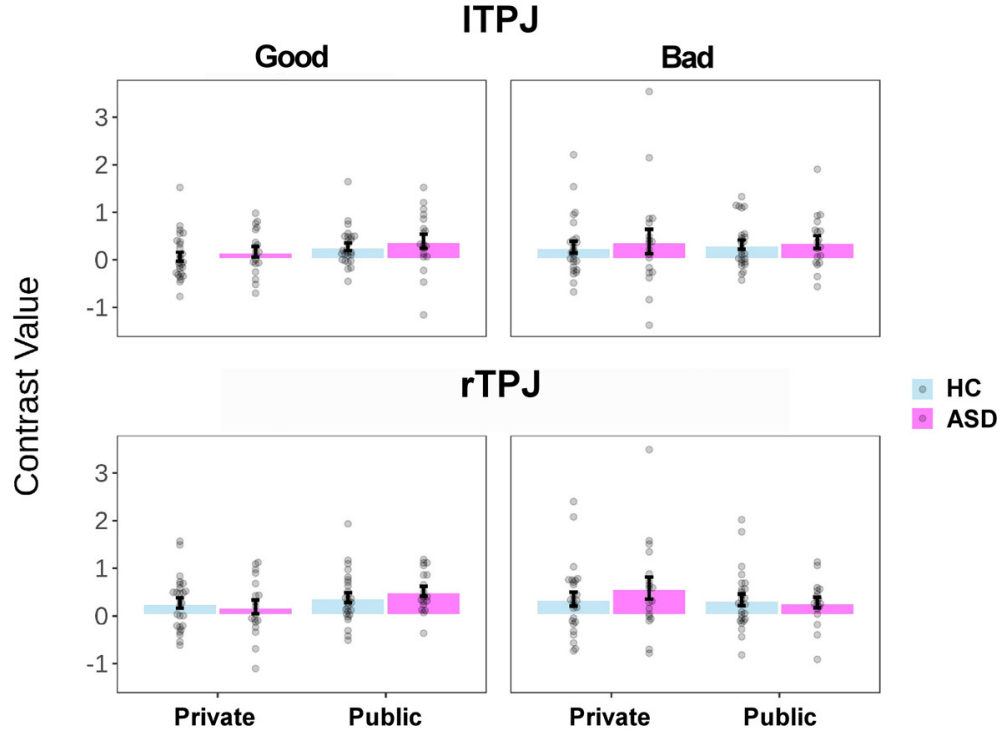

B

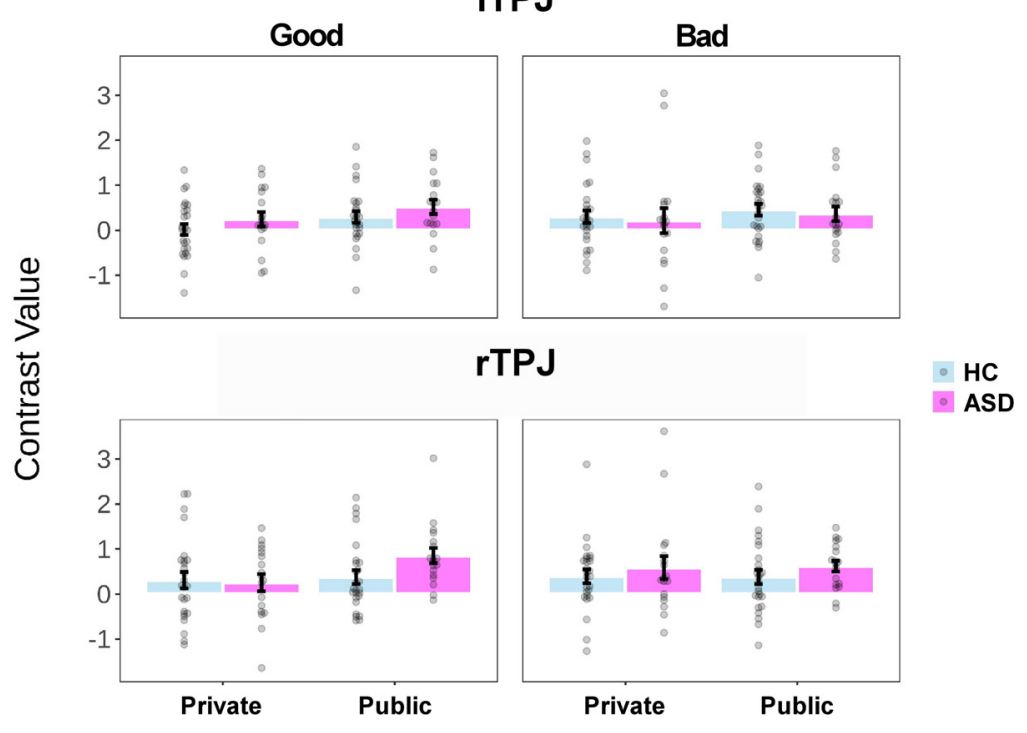

Figure 10. $\quad \boldsymbol{A}, \boldsymbol{B}$, Univariate results of TPJ in the HC and ASD groups using the parcellation-based mask $(\boldsymbol{A})$ and the coordinate-based mask $(\boldsymbol{B})$. For visualization, we extracted the mean activity (contrast value) of ITPJ and rTPJ from the corresponding masks as a function of group (ASD or HC), reputation (Private or Public), and context (Good or Bad). Each dot represents the data of a single participant. Error bars represent the SEM.

recent transcranial magnetic stimulation (TMS) study using a similar experimental paradigm has shown that disrupting rTPJ (vs sham) does not influence the audience effect on moral decisions in healthy individuals (Obeso et al., 2018). In addition, two earlier fMRI studies failed to find an increased activation of rTPJ in response to the presence (vs absence) of observers while healthy participants made charitable decisions (Izuma et al., 2010 b) or social evaluation (Izuma et al., 2010a). However, it is also worth noting that nonsignificant results do not necessarily reflect a true null effect (Makin and Xivry, 2019). Also, our RSA result suggests that multivoxel patterns of rTPJ represent the information of social reputation in healthy control subjects. Further studies are needed to clarify whether and how rTPJ plays a role in reputation-based decision-making.

Intriguingly, we did not observe a between-group difference of rTPJ in representing information about social reputation, although, as expected, a small but significant effect of social reputation on moral behaviors was observed only in healthy control subjects rather than in ASD participants. At first glance, this finding may seem at odds with the well established role of the rTPJ in mentalizing (and relevant social abilities) in both healthy participants (Hampton et al., 2008; Young et al., 2010; Carter et al., 2012; Morishima et al., 2012; Schurz et al., 2014; Hutcherson et al., 2015; Strombach et al., 2015; Hill et al., 2017; $\mathrm{Hu}$ et al., 2018; Qu et al., 2019) and ASD populations (Kana et al., 2009; Lombardo et al., 2011; Koster-Hale et al., 2013). These previous findings indicate that the alteration of ToM ability, reflected by the functional changes of rTPJ, determines the anomaly in moral behaviors in autistic cohorts. However, it should be noted that evidence also exists, revealing that ASD individuals may preserve some degree of ToM ability to guide their intent-based moral judgments. For instance, one study showed that autistic adults not only exhibit performance comparable to that of healthy control subjects in a false belief task but also report similar moral permissibility when judging intended harms with neutral outcomes (Moran et al., 2011). Another study even reported an increased sensitivity to intention during moral judgment in Asperger's syndrome compared with healthy control subjects (Channon et al., 2011). Consistent with these studies, our RSA results also suggest that the ability to represent the information on social reputation in rTPJ is partially intact in ASD participants. These findings indicate that the ability to infer and base moral judgments on intentionality may still be present in ASD individuals, and potentially explains why we did not observe a between-group difference of rTPJ in representing social reputation in our task. It has also been proposed that the method of inferring intentionality differs between autistic and neurotypical participants (Dempsey et al., 2020). Here, a reduced rTPJ representation similarity in ASD, unique to the moral context, explains that ASD individuals prioritize the negative consequences of an immoral action. This may block further recruitment of the intent-based system and thus lead to a lack of consideration for social reputation when making choices. Future studies may consider adopting tasks that involve both moral judgment and decision-making and implement noninvasive brain stimulation methods to target the rTPJ of ASD individuals to provide causal evidence for this possibility.

Despite the strengths of this study, there are two potential limitations. First, the sample size is relatively small for the ASD group, which could have lowered the statistical power 


\begin{tabular}{|c|c|c|c|c|c|c|c|c|}
\hline \multirow[b]{2}{*}{ Brain region } & \multirow[b]{2}{*}{ Hemisphere } & \multirow[b]{2}{*}{ Cluster size } & \multicolumn{3}{|l|}{$\mathrm{MNI}$} & \multirow[b]{2}{*}{ BA } & \multirow[b]{2}{*}{$t$ value } & \multirow[b]{2}{*}{$p(\mathrm{cl}-\mathrm{FWE})$} \\
\hline & & & $x$ & $y$ & $z$ & & & \\
\hline \multicolumn{9}{|l|}{ ASD } \\
\hline $\begin{array}{l}\quad \text { Cingulate gyrus/corpus callosum } \\
\mathrm{HC}>\text { ASD }\end{array}$ & L & 96 & -16 & 8 & 30 & & 4.99 & 0.162 \\
\hline \multicolumn{9}{|l|}{ Private $>$ public } \\
\hline Prec/SOG & L & 95 & -18 & -58 & 32 & 7 & 4.84 & 0.229 \\
\hline IPL/PoCG & L & 56 & -38 & -32 & 44 & 40 & 3.59 & 0.525 \\
\hline
\end{tabular}

We excluded trials that did not reach the behavioral criterion (i.e., those with a decision time $<200 \mathrm{~ms}$ or longer than mean \pm 3 SDs of that individual) or fMRI criterion (all trials in a run with an excessive head motion). Regions shown here met an uncorrected voxel-level threshold of $p<0.001$ with $k=50$. Coordinates shown here were based on the MNI coordinate system. L, Left; B, bilateral; BA, Brodmann Area; Cl-FWE, cluster-level FWE (corrected); CC, corpus callosum; CG, cingulate gyrus; IPL, inferior parietal lobule; PoCG: post-central gyrus; Prec, precuneus; SOG, superior occipital gyrus.

for the fMRI data analyses. Second, our sample has a relatively wide age range that covers the transition period from adolescence to early adulthood, during which time changes in sociocognitive processes and moral cognition continue to occur (Eisenberg and Morris, 2004; Blakemore and Mills, 2014; Kilford et al., 2016). Evidence indicates that mentalizing ability is still undergoing development in late adolescence (Dumontheil et al., 2010). More relevantly, previous studies have shown a distinct pattern in adolescents (vs adults) for prosocial behaviors (Padilla-Walker et al., 2018) or the susceptibility to the audience effect (Wolf et al., 2015). Importantly, these changes are considered to be crucially associated with the development of the social brain network in adolescence (Blakemore, 2008; Kilford et al., 2016). Taking TPJ as an example, evidence from brain imaging studies showed that both structural and functional features of this region vary during this transition period (Blakemore et al., 2007; Mills et al., 2014). Hence, the age-related heterogeneity of our sample may have had some impact on our results, although we controlled for age-related differences in our between-group analyses. Future studies with a larger sample or less age heterogeneity would allow more definite conclusions.

To conclude, the present study, combining computational modeling with multivariate fMRI analyses, uncovers the neurocomputational changes of the rTPJ during moral behaviors in autistic individuals. They are characterized not only by a lack of consideration for social reputation but also, more predominantly, by an increased sensitivity to the negative consequences caused by immoral actions. This difference in moral cognition and behaviors in ASD individuals is specifically associated with rTPJ and consists of a reduced capability to represent information concerning moral contexts. Our findings provide novel insights for a better understanding of the neurobiological basis underlying atypical moral behaviors in ASD individuals.

\section{References}

Ahn W-Y, Krawitz A, Kim W, Busmeyer JR, Brown JW (2011) A modelbased fMRI analysis with hierarchical Bayesian parameter estimation. J Neurosci Psychol Econ 4:95-110.

Ahn W-Y, Haines N, Zhang L (2017) Revealing neuro-computational mechanisms of reinforcement learning and decision-making with the hBayesDM package. Comput Psychiatr 1:24-57.

American Psychiatric Association (2013) Diagnostic and statistical manual of mental disorders: DSM-5, Ed 5. Arlington, VA: American Psychiatric Association.

Baron-Cohen S (2001) Theory of mind and autism: a review. Int Rev Res Ment Retard 23:169-184.
Baron-Cohen S, Leslie AM, Frith U (1985) Does the autistic child have a "theory of mind"? Cognition 21:37-46.

Bates D, Maechler M, Bolker B (2013) lme4: linear mixed-effects models using 44 classes. Vienna, Austria: R Foundation.

Bault N, Pelloux B, Fahrenfort JJ, Ridderinkhof KR, van Winden F (2015) Neural dynamics of social tie formation in economic decision-making. Soc Cogn Affect Neurosci 10:877-884.

Bellesi G, Vyas K, Jameel L, Channon S (2018) Moral reasoning about everyday situations in adults with autism spectrum disorder. Research in Autism Spectrum Disorders 52:1-11.

Blakemore S-J (2008) The social brain in adolescence. Nat Rev Neurosci 9:267-277.

Blakemore S-J, Mills KL (2014) Is adolescence a sensitive period for sociocultural processing? Annu Rev Psychol 65:187-207.

Blakemore S-J, Ouden D H, Choudhury S, Frith C (2007) Adolescent development of the neural circuitry for thinking about intentions. Soc Cogn Affect Neurosci 2:130-139.

Buon M, Dupoux E, Jacob P, Chaste P, Leboyer M, Zalla T (2013) The role of causal and intentional judgments in moral reasoning in individuals with high functioning autism. J Autism Dev Disord 43:458-470.

Burnham KP, Anderson DR (2004) Multimodel inference: understanding AIC and BIC in model selection. Sociol Methods Res 33:261-304.

Carter RM, Bowling DL, Reeck C, Huettel SA (2012) A distinct role of the temporal-parietal junction in predicting socially guided decisions. Science 337:109-111.

Channon S, Lagnado D, Fitzpatrick S, Drury H, Taylor I (2011) Judgments of cause and blame: sensitivity to intentionality in Asperger's syndrome. J Autism Dev Disord 41:1534-1542.

Crockett MJ (2016) How formal models can illuminate mechanisms of moral judgment and decision making. Curr Dir Psychol Sci 25:85-90.

Crockett MJ, Kurth-Nelson Z, Siegel JZ, Dayan P, Dolan RJ (2014) Harm to others outweighs harm to self in moral decision making. Proceedings of the National Academy of Sciences 111:17320-17325.

Crockett MJ, Siegel JZ, Kurth-Nelson Z, Dayan P, Dolan RJ (2017) Moral transgressions corrupt neural representations of value. Nature Neuroscience 20:879-885.

D'Cruz A-M, Ragozzino ME, Mosconi MW, Shrestha S, Cook EH, Sweeney JA (2013) Reduced behavioral flexibility in autism spectrum disorders. Neuropsychology 27:152-160.

Dempsey E, Moore C, Johnson S, Stewart S, Smith I (2020) Morality in autism spectrum disorder: a systematic review. Dev Psychopathol 32:10691085.

Dumontheil I, Apperly IA, Blakemore S-J (2010) Online usage of theory of mind continues to develop in late adolescence. Dev Sci 13:331-338.

Eisenberg N, Morris AS (2004) Moral cognitions and prosocial responding in adolescence. In: Handbook of adolescent psychology, Ed 2 (Lerner RM, Steinberg L, eds), pp 155-188. New York: Wiley.

Eklund A, Nichols TE, Knutsson H (2016) Cluster failure: why fMRI inferences for spatial extent have inflated false-positive rates. Proc Natl Acad Sci U S A 113:7900-7905.

Fadda R, Parisi M, Ferretti L, Saba G, Foscoliano M, Salvago A, Doneddu G (2016) Exploring the role of theory of mind in moral judgment: the case of children with autism spectrum disorder. Front Psychol 7:523. 
Fehr E, Schmidt KM (1999) A theory of fairness, competition, and cooperation. Q J Econ 114:817-868.

Fox J, Weisberg S, Adler D, Bates D, Baud-Bovy G, Ellison S, Firth D, Friendly M, Gorjanc G, Graves S (2016) Package "car". Vienna, Austria: R Foundation.

Frith U, Frith C (2011) Reputation management: in autism, generosity is its own reward. Curr Biol 21:R994-R995.

Gelman A, Rubin DB (1992) Inference from iterative simulation using multiple sequences. Stat Sci 7:457-472.

Gelman A, Carlin JB, Stern HS, Rubin DB (2014) Bayesian data analysis. Boca Raton, FL: Chapman \& Hall/CRC.

Gelman A, Lee D, Guo J (2015) Stan: A Probabilistic Programming Language for Bayesian Inference and Optimization. Journal of Educational and Behavioral Statistics 40:530-543.

Haley KJ, Fessler DMT (2005) Nobody's watching?: Subtle cues affect generosity in an anonymous economic game. Evol Hum Behav 26:245-256.

Hampton AN, Bossaerts P, O'Doherty JP (2008) Neural correlates of mentalizing-related computations during strategic interactions in humans. Proc Natl Acad Sci U S A 105:6741-6746.

Hebart MN, Baker CI (2018) Deconstructing multivariate decoding for the study of brain function. Neuroimage 180:4-18.

Hill CA, Suzuki S, Polania R, Moisa M, O’Doherty JP, Ruff CC (2017) A causal account of the brain network computations underlying strategic social behavior. Nat Neurosci 20:1142-1149.

Hu Y, He L, Zhang L, Wölk T, Dreher JC, Weber B (2018) Spreading inequality: neural computations underlying paying-it-forward reciprocity. Soc Cogn Affect Neurosci 13:578-589.

Hutcherson C, Bushong B, Rangel A (2015) A neurocomputational model of altruistic choice and its implications. Neuron 87:451-462.

Izuma K (2012) The social neuroscience of reputation. Neurosci Res 72:283288.

Izuma K, Saito DN, Sadato N (2008) Processing of social and monetary rewards in the human striatum. Neuron 58:284-294.

Izuma K, Saito DN, Sadato N (2010a) The roles of the medial prefrontal cortex and striatum in reputation processing. Soc Neurosci 5:133-147.

Izuma K, Saito DN, Sadato N (2010b) Processing of the incentive for social approval in the ventral striatum during charitable donation. J Cogn Neurosci 22:621-631.

Izuma K, Matsumoto K, Camerer CF, Adolphs R (2011) Insensitivity to social reputation in autism. Proc Natl Acad Sci U S A 108:17302-17307.

Kana RK, Keller TA, Cherkassky VL, Minshew NJ, Just MA (2009) Atypical frontal-posterior synchronization of theory of mind regions in autism during mental state attribution. Soc Neurosci 4:135-152.

Kilford EJ, Garrett E, Blakemore SJ (2016) The development of social cognition in adolescence: an integrated perspective. Neurosci Biobehav Rev 70:106-120.

Konovalov A, Hu J, Ruff CC (2018) Neurocomputational approaches to social behavior. Curr Opin Psychol 24:41-47.

Koster-Hale J, Saxe R, Dungan J, Young LL (2013) Decoding moral judgments from neural representations of intentions. Proc Natl Acad Sci U S A 110:5648-5653.

Kriegeskorte N, Mur M, Bandettini PA (2008) Representational similarity analysis-connecting the branches of systems neuroscience. Front Syst Neurosci 2:4.

Lombardo MV, Chakrabarti B, Bullmore ET, Baron-Cohen S, Consortium MA (2011) Specialization of right temporo-parietal junction for mentalizing and its relation to social impairments in autism. Neuroimage 56:1832-1838.

Lopez-Persem A, Rigoux L, Bourgeois-Gironde S, Daunizeau J, Pessiglione M (2017) Choose, rate or squeeze: comparison of economic value functions elicited by different behavioral tasks. PLoS Comput Biol 13: e1005848.

Luke SG (2017) Evaluating significance in linear mixed-effects models in R. Behav Res Methods 49:1494-1502.

Makin TR, Xivry JJOD (2019) Ten common statistical mistakes to watch out for when writing or reviewing a manuscript. Elife 8:e48175.
Margoni F, Surian L (2016) Mental state understanding and moral judgment in children with autistic spectrum disorder. Front Psychol 7:1478.

Mills KL, Lalonde F, Clasen LS, Giedd JN, Blakemore S-J (2014) Developmental changes in the structure of the social brain in late childhood and adolescence. Soc Cogn Affect Neurosci 9:123-131.

Moran JM, Young LL, Saxe R, Lee SM, O’Young D, Mavros PL, Gabrieli JD (2011) Impaired theory of mind for moral judgment in high-functioning autism. Proc Natl Acad Sci U S A 108:2688-2692.

Morishima Y, Schunk D, Bruhin A, Ruff CC, Fehr E (2012) Linking brain structure and activation in temporoparietal junction to explain the neurobiology of human altruism. Neuron 75:73-79.

Norman KA, Polyn SM, Detre GJ, Haxby JV (2006) Beyond mind-reading: multi-voxel pattern analysis of fMRI data. Trends Cogn Sci 10:424-430.

Obeso I, Moisa M, Ruff CC, Dreher J-C (2018) A causal role for right temporo-parietal junction in signaling moral conflict. eLife 7:e40671.

Padilla-Walker LM, Carlo G, Memmott-Elison MK (2018) Longitudinal change in adolescents' prosocial behavior toward strangers, friends, and family. J Res Adolesc 28:698-710.

Pantelis PC, Byrge L, Tyszka JM, Adolphs R, Kennedy DP (2015) A specific hypoactivation of right temporo-parietal junction/posterior superior temporal sulcus in response to socially awkward situations in autism. Soc Cogn Affect Neurosci 10:1348-1356.

Popal HS, Olson IR Wang Y (2019) A Guide to Representational Similarity Analysis for Social Neuroscience. Social Cognitive and Affective Neuroscience 14:1243-1253.

Qu C, Météreau E, Butera L, Villeval MC, Dreher J-C (2019) Neurocomputational mechanisms at play when weighing concerns for extrinsic rewards, moral values, and social image. PLoS Biol 17:e3000283.

Qu C, Hu Y, Tang Z, Derrington E, Dreher JC (2020) Neurocomputational mechanisms underlying immoral decisions benefiting self or others. Soc Cogn Affect Neurosci 15:135-149.

R Core Team (2014) R: a language and environment for statistical computing. Vienna, Austria: R Foundation.

Salvano-Pardieu V, Blanc R, Combalbert N, Pierratte A, Manktelow K, Maintier C, Lepeltier S, Gimenes G, Barthelemy C, Fontaine R (2016) Judgment of blame in teenagers with Asperger's syndrome. Think Reason 22:251-273.

Schaafsma SM, Pfaff DW, Spunt RP, Adolphs R (2015) Deconstructing and reconstructing theory of mind. Trends Cogn Sci 19:65-72.

Schaller UM, Biscaldi M, Fangmeier T, van Elst LT, Rauh R (2019) Intuitive moral reasoning in high-functioning autism spectrum disorder: a matter of social schemas? J Autism Dev Disord 49:1807-1824.

Schurz M, Radua J, Aichhorn M, Richlan F, Perner J (2014) Fractionating theory of mind: a meta-analysis of functional brain imaging studies. Neurosci Biobehav Rev 42:9-34.

Strombach T, Weber B, Hangebrauk Z, Kenning P, Karipidis II, Tobler PN, Kalenscher T (2015) Social discounting involves modulation of neural value signals by temporoparietal junction. Proc Natl Acad Sci U S A 112:1619-1624.

Tusche A, Böckler A, Kanske P, Trautwein F-M, Singer T (2016) Decoding the charitable brain: empathy, perspective taking, and attention shifts differentially predict altruistic giving. J Neurosci 36:4719-4732.

Watanabe T, Lawson RP, Walldén YSE, Rees G (2019) A neuroanatomical substrate linking perceptual stability to cognitive rigidity in autism. J Neurosci 39:6540-6554.

Wickham H (2016) ggplot2: elegant graphics for data analysis. Cham, Switzerland: Springer.

Wolf LK, Bazargani N, Kilford EJ, Dumontheil I, Blakemore SJ (2015) The audience effect in adolescence depends on who's looking over your shoulder. J Adolesc 43:5-14.

Young L, Cushman F, Hauser M, Saxe R (2007) The neural basis of the interaction between theory of mind and moral judgment. Proc Natl Acad Sci U S A 104:8235-8240.

Young L, Camprodon JA, Hauser M, Pascual-Leone A, Saxe R (2010) Disruption of the right temporoparietal junction with transcranial magnetic stimulation reduces the role of beliefs in moral judgments. Proc Natl Acad Sci U S A 107:6753-6758. 